\title{
Determination of the binding
} energies of the np Rydberg states of $\mathrm{H} 2, \mathrm{HD}$, and $\mathrm{D} 2$ from highresolution spectroscopic data by multichannel quantum-defect theory

\section{Journal Article}

Author(s):

Sprecher, Daniel; Jungen, Christian; Merkt, Frédéric

Publication date:

2014-03

Permanent link:

https://doi.org/10.3929/ethz-b-000081465

Rights / license:

In Copyright - Non-Commercial Use Permitted

Originally published in:

The Journal of Chemical Physics 140(10), https://doi.org/10.1063/1.4866809

Funding acknowledgement:

146759 - Rydberg states, VUV laser spectroscopy and photoionization dynamics (SNF) 
This article may be downloaded for personal use only. Any other use requires prior permission of the author and AIP Publishing.

The following article appeared in J. Chem. Phys. 140, 064304 (2014) and may be found at http://dx.doi.org/10.1063/1.4864002. 


\section{An accurate quantum-defect model for the triplet $n p$ Rydberg states of $\mathbf{H e}_{2}$}

Daniel Sprecher, ${ }^{1}$ Jinjun Liu, ${ }^{1,}$ a) Tobias Krähenmann, ${ }^{1}$ Martin Schäfer, ${ }^{1}$ and Frédéric Merkt $^{1, b)}$

Laboratorium für Physikalische Chemie, ETH-Zürich, 8093 Zürich, Switzerland

(Dated: 19 November 2019)

Photoionization spectra and Rydberg-state-resolved threshold-ionization spectra of triplet $n p$ Rydberg states of ${ }^{4} \mathrm{He}_{2}$ located in the vicinity of the $X^{+2} \Sigma_{u}^{+}\left(v^{+}=0\right)$ ionization threshold were recorded from the $2 s \sigma a^{3} \Sigma_{u}^{+}$metastable state. An accuracy of $0.01 \mathrm{~cm}^{-1}$ was achieved for the experimental term values of the observed Rydberg states. The data were combined with spectroscopic data on low-lying triplet $n p$ and nf Rydberg states from the literature to derive energy- and internuclear-distancedependent eigenquantum-defect parameters of multichannel quantum-defect theory (MQDT). The MQDT calculations reproduce the experimental data within their experimental uncertainties and enabled the derivation of potential-energy curves for the lowest triplet $p$ Rydberg states $(n=2-5)$ of $\mathrm{He}_{2}$. The eigenquantum-defect parameters describing the $p-f$ interaction were found to be larger than 0.002 at the energies corresponding to the high- $n$ Rydberg states, so that the $p$ - $f$ interaction plays an important role in the autoionization dynamics of $n p$ Rydberg states with $v^{+}=0$. By extrapolating the experimental term values of triplet $n p$ Rydberg states of ${ }^{4} \mathrm{He}_{2}$ in the range of principal quantum number $n$ between 87 and 110, the positions of the $\left(v^{+}=0, N^{+}=3\right)$ and $\left(v^{+}=0, N^{+}=5\right)$ levels of the ground state of ${ }^{4} \mathrm{He}_{2}^{+}$ were determined to lie $70.937(3) \mathrm{cm}^{-1}$ and $198.369(6) \mathrm{cm}^{-1}$, respectively, above the $\left(v^{+}=0, N^{+}=1\right)$ ground rotational level.

PACS numbers: 33.80.Rv, 33.80.Eh, 33.60.+q, 33.20.Wr, 33.20.Tp, 33.20.Sn, 33.15.Ry Keywords: helium dimer, Rydberg-state-resolved threshold-ionization spectroscopy, rotational and vibrational channel interactions, $p-f$ interaction, field-induced autoionization, $\mathrm{He}_{2}^{+}$

\footnotetext{
a) Present address: Department of Chemistry and Conn Center for Renewable Energy Research, University of Louisville, Louisville, Kentucky 40292, USA

b)Electronic mail: frederic.merkt@phys.chem.ethz.ch
} 


\section{INTRODUCTION}

Atomic helium has the lowest polarizability ${ }^{1}\left(0.205 \AA^{3}\right)$ and the largest ionization energy ${ }^{2}$ (198310.6664(2) $\left.\mathrm{cm}^{-1}\right)$ of all neutral elements and is chemically very inert. ${ }^{3}$ The potentialenergy well of $\mathrm{He}_{2}$ in the $X^{1} \Sigma_{g}^{+}$ground state is so shallow that it only supports a single quantum state $(v=0, N=0)$ for ${ }^{4} \mathrm{He}_{2}$ with a dissociation energy $D_{0}\left({ }^{4} \mathrm{He}_{2}\right)=0.00112(2) \mathrm{cm}^{-1} \cdot{ }^{4-6}$ The $X^{+2} \Sigma_{u}^{+}$ground state of $\mathrm{He}_{2}^{+}$corresponds to the electronic configuration $\left(1 s \sigma_{g}\right)^{2}\left(1 s \sigma_{u}\right)^{1}$. $\mathrm{He}_{2}^{+}$therefore has a covalent bond of order $1 / 2$ with a Born-Oppenheimer binding energy $D_{e}\left(\mathrm{He}_{2}^{+}\right)=19954.583 \mathrm{~cm}^{-1} \cdot{ }^{7}$

The rovibrational energy levels of $\mathrm{He}_{2}^{+}$serve as benchmark quantities for ab initio calculations of three-electron systems. ${ }^{7-16}$ In contrast to the numerous theoretical studies reported on $\mathrm{He}_{2}^{+}$, only a few experimental studies have been carried out. For the main naturally occurring isotopomer, ${ }^{4} \mathrm{He}_{2}^{+}$, no electric-dipole-allowed rotational and vibrational spectra exist. $\mathrm{Yu}$ and $\mathrm{Wing}^{17}$ observed nine transitions in the fundamental vibrational band of the heteronuclear ${ }^{3} \mathrm{He}^{4} \mathrm{He}^{+}$isotopomer, which exhibits a small electric dipole moment. Carrington et al. ${ }^{11}$ measured the frequency of seven electronic transitions from vibrationally highly excited levels of the $X^{+2} \Sigma_{u}^{+}$ground state of ${ }^{4} \mathrm{He}_{2}^{+}$to the weakly bound $A^{+2} \Sigma_{g}^{+}$first excited state. Raunhardt et al. ${ }^{18}$ determined rotational constants for the lowest three vibrational levels of ${ }^{4} \mathrm{He}_{2}^{+}$and observed the rotational structure of the ground state of ${ }^{3} \mathrm{He}_{2}^{+}$by pulsedfield-ionization zero-kinetic-energy (ZEKE) photoelectron spectroscopy of metastable $\mathrm{He}_{2}$. Experimental results obtained by Hardy and coworkers ${ }^{19}$ on the rotational structure of ${ }^{4} \mathrm{He}_{2}^{+}$ have been questionned because their analysis is based on the observation of states which do not fulfill the generalized Pauli principle (see also discussion in Refs. 18, 20, and 21).

Ginter and coworkers ${ }^{22-26}$, and also Raunhardt et al. ${ }^{18}$, measured the term values of triplet $n p$ Rydberg states of $\mathrm{He}_{2}$ and obtained rovibrational energy spacings in $\mathrm{He}_{2}^{+}$by extrapolation to the series limits using MQDT. Their model included rotational channel interactions and the dependence of the eigenquantum-defect parameters on the energy, the rotational quantum number, and the vibrational quantum number. The accuracy of the extrapolated energies presented in Refs. 23 and 18 was limited to about $0.1 \mathrm{~cm}^{-1}$. In the experiments presented in this article, an accuracy of $0.01 \mathrm{~cm}^{-1}$ was achieved in the determination of the term values of high- $n p$ Rydberg states of ${ }^{4} \mathrm{He}_{2}$ and in the extrapolation of the observed Rydberg series to their limits using an extended MQDT model. This model includes internuclear- 
distance- and energy-dependent eigenquantum-defect functions and considers the effects of the $p-f$ interaction, which is known to induce perturbations in the structure of the $n p$ and

nf Rydberg states of $\mathrm{He}_{2} \cdot{ }^{23,27}$ Using this model, a global description of the triplet $n p$ Rydberg states of $\mathrm{He}_{2}$ was achieved in a fit of the relevant MQDT parameters to spectroscopic data available in the literature. ${ }^{22-29}$ With this improved model, the positions of all known triplet $n p$ Rydberg states can be calculated within their experimental uncertainties, BornOppenheimer potential-energy curves of the 2-5p Rydberg states were derived, and the corresponding leading adiabatic corrections were estimated.

\section{EXPERIMENT}

\section{A. Production and photoionization spectroscopy of $\mathrm{He}_{2}$}

A schematic diagram of the experimental chamber is shown in Fig. 1. It consists of a gas source chamber and a photoexcitation/detection chamber separated by a skimmer with an orifice diameter of $1 \mathrm{~mm}$. A supersonic beam containing ${ }^{4} \mathrm{He}_{2}$ in the $2 s \sigma a^{3} \Sigma_{u}^{+}$metastable state (referred to as $\mathrm{He}_{2}^{*}$ hereafter) was produced from atomic helium by striking an electric discharge at the orifice of a pulsed nozzle. The discharge design has been described in detail previously. ${ }^{18}$ The phosphorescence-limited lifetime of $\mathrm{He}_{2}^{*}$ is $13(2) \mathrm{s},{ }^{30}$ which is much longer than the time $(\sim 150 \mu \mathrm{s})$ required for the metastable molecules to reach the photoexcitation region. The photoexcitation region was surrounded by a cylindrically symmetric stack of resistively coupled metallic electrodes designed for the application of homogeneous electric fields and by two concentric magnetic shields made of mu-metal to suppress stray magnetic fields. Differential pumping resulted in background pressures of $10^{-4}$ mbar and $5 \times 10^{-7} \mathrm{mbar}$ in the gas source chamber and the photoexcitation/detection chamber, respectively.

Triplet $n p$ Rydberg states of $\mathrm{He}_{2}$ were excited from $\mathrm{He}_{2}^{*}$ using the third harmonic (wavelength $292 \mathrm{~nm}$, pulse length $30 \mathrm{~ns}$, bandwidth $<0.001 \mathrm{~cm}^{-1}$, pulse energy $\left.\sim 150 \mu \mathrm{J}\right)$ of the output of a Ti:Sa laser system described in Ref. 31. A pulsed homogeneous electric field (amplitude $128 \mathrm{~V} / \mathrm{cm}$ ) was generated $200 \mathrm{~ns}$ after laser excitation by applying a pulsed voltage across the electrode stack. The electric field was used to ionize Rydberg states and its polarity was chosen such that the positively charged ions were accelerated towards a microchannel-plate (MCP) detector. The ion signal corresponding to the time of flight of 


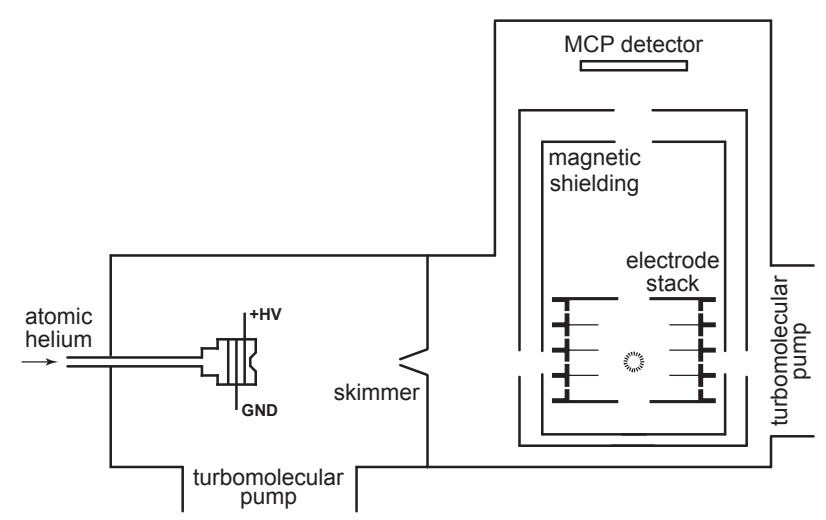

FIG. 1. Schematic diagram of the vacuum chambers used in the experiments. The photoexcitation region is marked with a circle.

the $\mathrm{He}_{2}^{+}$ions was integrated and monitored as a function of the laser wave number.

\section{B. Rydberg-state-resolved threshold-ionization spectroscopy}

$\mathrm{He}_{2}^{*}$ molecules in many different rovibrational states are produced in the electric discharge and the Rydberg series excited from these states and observed in the photoionization (PI) spectra overlap, which makes unambiguous assignments very difficult. By changing to the detection scheme of Rydberg-state-resolved threshold-ionization (RSR-TI) spectroscopy, ${ }^{32}$ which restricts the detection to electrons produced by field ionization of long-lived Rydberg states, the spectral congestion could be considerably reduced. In RSR-TI spectroscopy, a longer delay ( $2 \mu \mathrm{s}$ in the present case) and an electric field of smaller amplitude $(12.8 \mathrm{~V} / \mathrm{cm})$ and opposite polarity compared to PI spectroscopy are used to field-ionize the Rydberg states. The spectra are recorded by monitoring the electron signal detected on the MCP detector as a function of the laser wave number. Electrons originating from direct photoionization or from autoionizing Rydberg states with lifetimes shorter than $2 \mu$ s leave the photoexcitation region before the electric-field pulse is applied and are not detected. RSRTI spectroscopy was already applied in our group to study high- $n$ Rydberg states of $\mathrm{N}_{2},{ }^{32}$ $\mathrm{NH}_{3},{ }^{33}$ and $\mathrm{Kr}^{34}$ and the corresponding cationic states at high resolution. 
TABLE I. List of contributions to the absolute and relative uncertainties in the determination of the transition wave numbers. All values are given in units of $\mathrm{cm}^{-1}$.

\begin{tabular}{|c|c|}
\hline Source of uncertainty & Uncertainty in UV \\
\hline \multicolumn{2}{|c|}{ Calibration of absolute wave numbers } \\
\hline Positions of iodine reference lines ${ }^{35}$ & 0.009 \\
\hline Doppler shift & 0.004 \\
\hline Frequency shift in the Ti:Sa amplifier & $<0.001$ \\
\hline Pressure shift & $<0.001$ \\
\hline Total absolute uncertainty ${ }^{\mathrm{a}}$ & 0.010 \\
\hline \multicolumn{2}{|c|}{ Calibration of relative wave numbers } \\
\hline Calibration of relative laser frequency & $0.006^{\mathrm{b}}$ \\
\hline Determination of the line centers & 0.001 \\
\hline Stark shifts & $<0.001^{\mathrm{c}}$ \\
\hline Total relative uncertainty ${ }^{\mathrm{a}}$ & $0.006^{\mathrm{b}}$ \\
\hline
\end{tabular}

${ }^{a}$ Determined by summation of all contributions in quadrature.

b Assuming that the transitions are separated by $\sim 12 \mathrm{~cm}^{-1}$. Because the relative laser frequency is determined by extrapolation, the uncertainty is proportional to the interval (see text).

c This value was estimated for $n p$ Rydberg states with $n<50$. At higher values of $n$, the dc Stark shifts can be larger.

\section{Wave-number calibration}

For the absolute wave-number calibration, iodine absorption spectra were recorded simultaneously with the PI and RSR-TI spectra of $\mathrm{He}_{2}^{*}$ using part of the fundamental cw radiation near $876 \mathrm{~nm}$. The transition wave numbers of selected lines tabulated in the iodine atlas of Gerstenkorn and coworkers ${ }^{35}$ served as reference, resulting in an estimated uncertainty in the absolute wave-number calibration of $0.003 \mathrm{~cm}^{-1}$ in the fundamental near-infrared radiation and $0.009 \mathrm{~cm}^{-1}$ in the upconverted ultraviolet (UV) radiation. Although the iodine cell was heated up to $800^{\circ} \mathrm{C}$, the signal was too weak to record saturated Doppler-free $\mathrm{I}_{2}$ spectra, which would have allowed for a more accurate wave-number calibration.

Relative laser wave numbers were determined using the transmission signal through a frequency-stabilized high-finesse Fabry-Pérot etalon. The free spectral range (FSR) of this etalon was determined in Ref. 36 to be 149.969(1) MHz using radiation at $792 \mathrm{~nm}$. This value was found to also be valid within $0.03 \mathrm{MHz}$ at $876 \mathrm{~nm}$ using iodine absorption spectroscopy as described above. A FSR of $149.969(30) \mathrm{MHz}$ was therefore used in the present study. Because the relative wave-number calibration is limited by the uncertainty of the FSR of the etalon, the uncertainty in the relative positions of two transitions is proportional to 


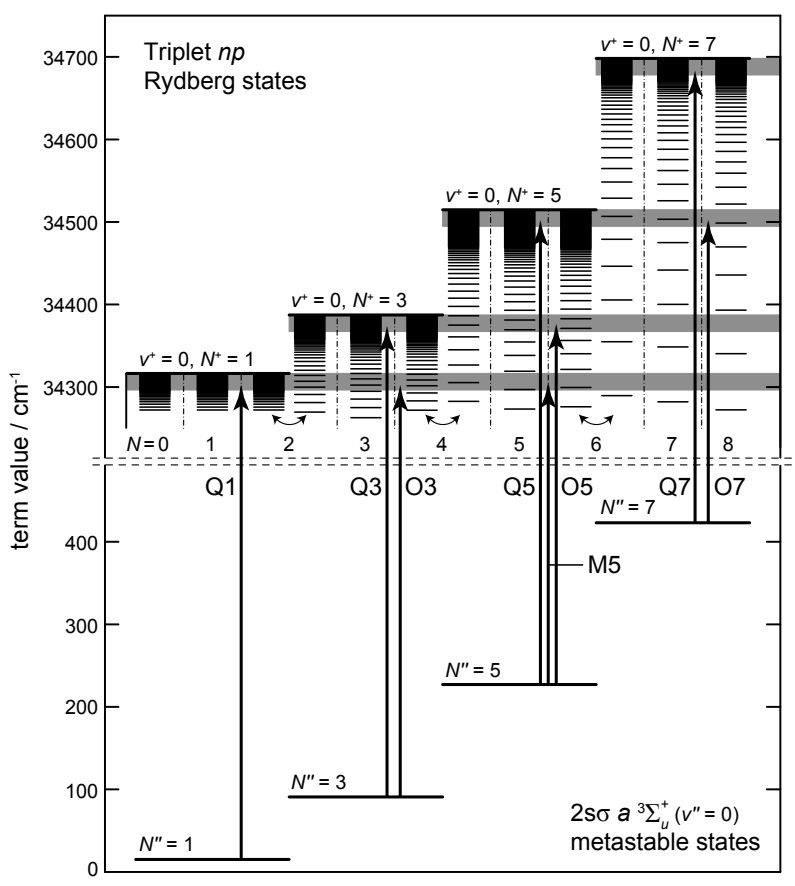

FIG. 2. Energy-level diagram of the rotational levels of ${ }^{4} \mathrm{He}_{2}^{*}$ and the triplet $n p$ Rydberg states of ${ }^{4} \mathrm{He}_{2}$. The regions shaded in gray are those probed by RSR-TI spectroscopy using electric-field pulses of $12.8 \mathrm{~V} / \mathrm{cm}$. The vertical arrows designate the eight spectral regions investigated in the present study. The origin of the wave-number scale was placed at the position of the origin of the $2 \mathrm{~s} \sigma a^{3} \Sigma_{u}^{+}$state which corresponds to the position of the Pauli-forbidden $\left(v^{\prime \prime}=0, N^{\prime \prime}=0, J^{\prime \prime}=1\right)$ level.

their separation. For typical separations of less than $12 \mathrm{~cm}^{-1}$, the relative uncertainty in the determination of transition wave numbers is estimated to be less than $0.006 \mathrm{~cm}^{-1}$. All contributions to the estimated total absolute and relative uncertainties of $0.01 \mathrm{~cm}^{-1}$ and $0.006 \mathrm{~cm}^{-1}$, respectively, are listed in Table I. The systematic uncertainties resulting from Doppler shifts, pressure shifts, and Stark shifts were estimated as described in Ref. 37.

\section{RESULTS}

\section{A. Observed transitions and their assignment}

A schematic energy level diagram of the initial metastable states and final triplet $n p$ Rydberg states investigated in the present study is depicted in Fig. 2. When neglecting channel interactions, the spectral positions $\tilde{\nu}$ of Rydberg states are well described by the 
Rydberg formula

$$
\tilde{\nu}=\frac{E^{+}\left(v^{+}, N^{+}\right)}{h c}-\frac{\mathcal{R}_{\mathrm{He}_{2}}}{(n-\bar{\mu})^{2}},
$$

where $h$ is Planck's constant, $c$ is the speed of light in vacuum, $E^{+}\left(v^{+}, N^{+}\right)$are the energies of the rovibrational ionization thresholds, $\mathcal{R}_{\mathrm{He}_{2}}=109729.796 \mathrm{~cm}^{-1}$ is the mass-corrected Rydberg constant, and $\bar{\mu}$ is the effective quantum defect. Rydberg states with even values of $N^{+}$do not exist because their wave function would be antisymmetric with respect to the permutation of the two bosonic ${ }^{4} \mathrm{He}^{2+}$ nuclei and therefore violate the generalized Pauli principle. For the same reason, rotational levels of the metastable state with even values of $N^{\prime \prime}$ do not exist.

The pulsed electric field used to ionize the Rydberg states when recording the RSR-TI spectra had an amplitude of $12.8 \mathrm{~V} / \mathrm{cm}$, which lowers the ionization thresholds by $\sim 20 \mathrm{~cm}^{-1}$ and restricts the energetic detection windows to the regions marked by areas shaded in gray in Fig. 2. Eight spectral regions, marked by vertical arrows in Fig. 2, were investigated at high spectral resolution. These regions are identified using the labels Q1, Q3, Q5, Q7, O3, O5, O7, and M5 which designate the different ionization thresholds according to the change in the rotational quantum number $\Delta N=N^{+}-N^{\prime \prime}$ and the value of $N^{\prime \prime}$. Regions which correspond to $\Delta N=0,-2$, and -4 transitions are labeled using the letters ' $\mathrm{Q}$ ', ' $\mathrm{O}$ ', and ' $\mathrm{M}$ ', respectively. The present investigation was restricted to transitions with $v^{\prime \prime}=v^{+}=0$. For a given value of $N^{+}$, three $n p$ Rydberg series can be observed with $N=N^{+}-1, N^{+}$, and $N^{+}+1$. Throughout this article, the notation $N^{\prime \prime} \rightarrow n \ell N_{N}^{+}$is used to label transitions from the rotational levels of the $2 \mathrm{~s} \sigma a^{3} \Sigma_{u}^{+}\left(v^{\prime \prime}=0, N^{\prime \prime}\right)$ metastable states to triplet $n \ell N_{N}^{+}\left(v^{+}=0\right)$ Rydberg states.

As illustrations of the data obtained experimentally, representative sections of the PI and RSR-TI spectra recorded in regions Q5 and O7 are depicted in panels (a) and (b) of Fig. 3, respectively. The corresponding etalon transmission spectra and iodine absorption spectra used for calibration are also displayed. The intensities of the etalon transmission lines fluctuate because the step size of the laser was comparable to their linewidth. In the PI spectrum (top trace in Fig. 3a), the expected transitions from the $\left(v^{\prime \prime}=0, N^{\prime \prime}=5\right)$ level of the metastable state to high- $n p$ Rydberg states with $N^{+}=5$ (see assignment bars) are obscured by transitions from higher-lying rovibrational levels of the metastable state to autoionizing low- $n p$ Rydberg states. In the corresponding RSR-TI spectrum, only the $n p 5_{6}$ 


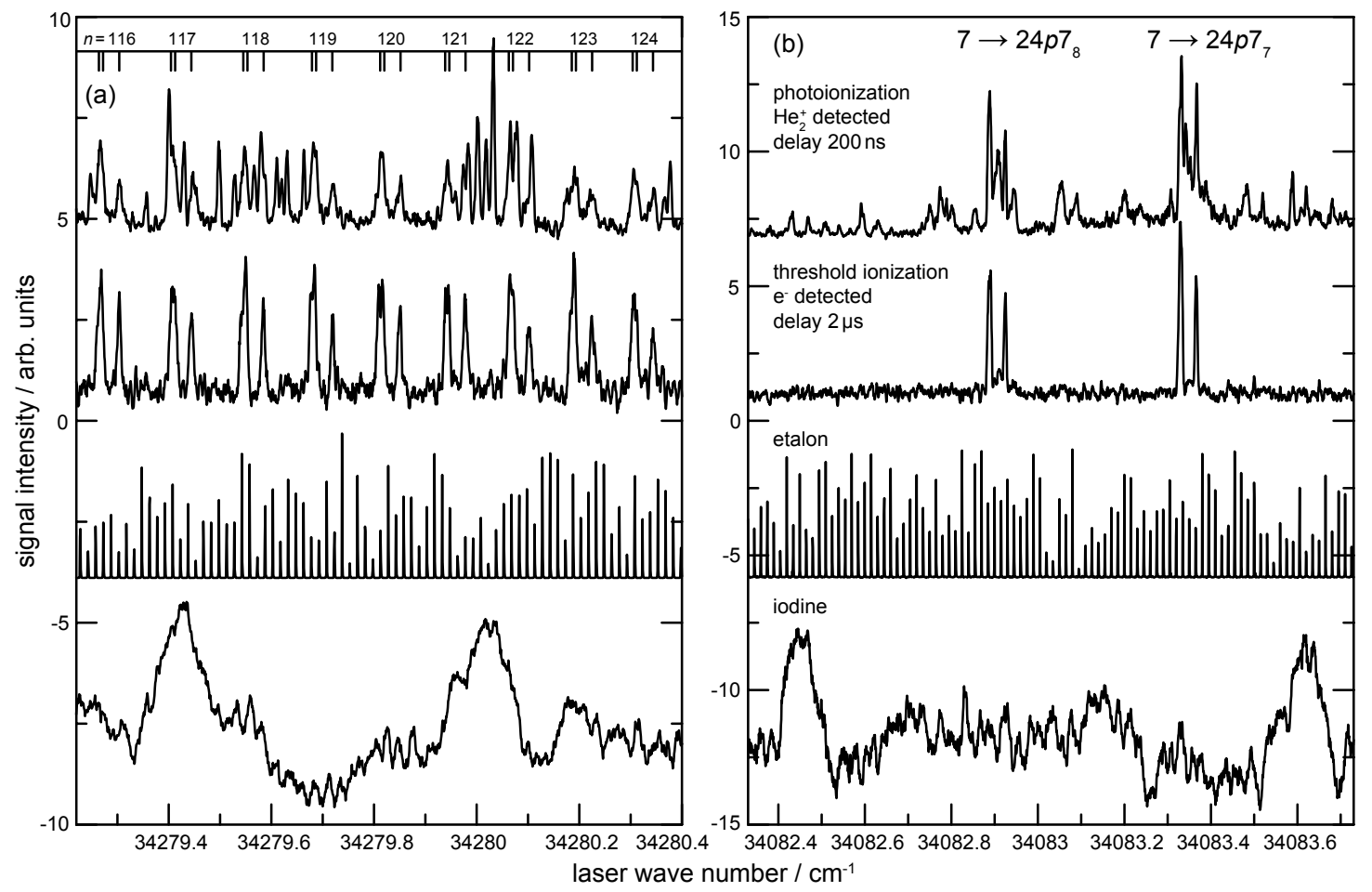

FIG. 3. Comparison between the PI spectra (top trace) and the RSR-TI spectra (second trace from top) recorded in (a) region Q5 and (b) region O7 (see Fig. 2 for the definition of these regions). Simultaneously with the $\mathrm{He}_{2}$ spectra, etalon traces (third trace from top) and iodine spectra (bottom trace) were recorded for the relative and absolute frequency calibrations, respectively (shifted along the vertical axis for clarity). In panel (a) the transitions are labeled with the principal quantum number $n$ of the $5 \rightarrow n p 5_{6}$ series.

Rydberg series is visible because the delayed detection of electrons (delay $2 \mu \mathrm{s}$ ) prevents Rydberg states with lifetimes $\ll 2 \mu$ s from being detected. The splitting observed for each $5 \rightarrow n p 5_{6}$ transition originates from the fine structure of the initial metastable state, as discussed in Section III B. The $n p 5_{4}$ and $n p 5_{5}$ Rydberg series are not observed in region Q5 of the RSR-TI spectrum, for reasons that are discussed in Section IIIF.

The PI and RSR-TI spectra in region O7 displayed in Fig. 3b are dominated by the $N=7$ and $N=8$ orbital fine-structure components of the $7 \rightarrow n p 7_{N}$ series which were assigned on the basis of the calculations presented in Ref. 18. The $24 p 7_{N}$ Rydberg states are located about $192 \mathrm{~cm}^{-1}$ below the $N^{+}=7$ ionization threshold (see Fig. 2) and it may seem surprising, at first sight, that these states are at all field ionized by the $12.8 \mathrm{~V} / \mathrm{cm}$ pulsed electric field used to record the RSR-TI spectrum. The observation can be explained by the mechanism of field-induced rotational autoionization: ${ }^{38,39}$ The $24 p 7_{N}$ Rydberg states are located about $9 \mathrm{~cm}^{-1}$ below the $N^{+}=5$ ionization threshold. The pulsed electric field 

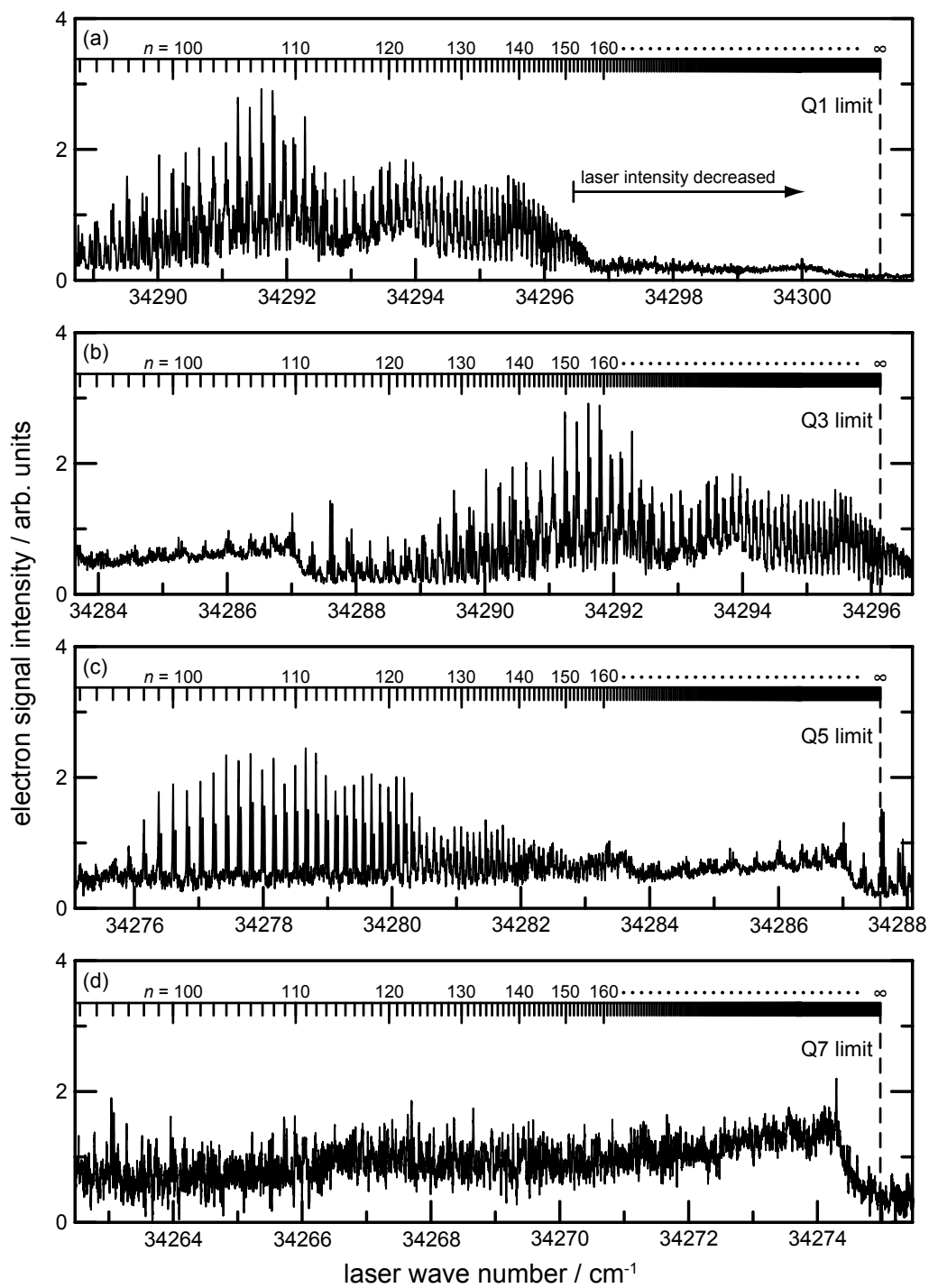

FIG. 4. RSR-TI spectra of ${ }^{4} \mathrm{He}_{2}$ recorded in regions (a) Q1, (b) Q3, (c) Q5, and (d) Q7 (see Fig. 2 for the definition of these regions).

leads to the ionization of the $24 p 7_{N}$ Rydberg states because it makes the $N^{+}=5$ ionization continuum energetically accessible and also because it facilitates rotational autoionization. ${ }^{38}$

No transitions to $N^{+}=5$ Rydberg states are observed in region O7 because the excitation efficiency from the initial $N^{\prime \prime}=7$ state is too low. The dominant Rydberg series are those with $N^{+}=N^{\prime \prime}$ and transitions corresponding to $N^{+}=N^{\prime \prime} \pm 2, N^{\prime \prime} \pm 4, \ldots$ make no significant contribution to the spectra (see discussion in Ref. 18). This situation is also encountered in $n p$ Rydberg series of $\mathrm{H}_{2}$ (see, e.g., Ref. 40) and $\mathrm{N}_{2}$ (see, e.g., Ref. 41) when excitation is from an initial orbital of dominant $s$ character. ${ }^{42}$

The RSR-TI spectra recorded in regions Q1-Q7 are presented in Fig. 4. Transitions to 


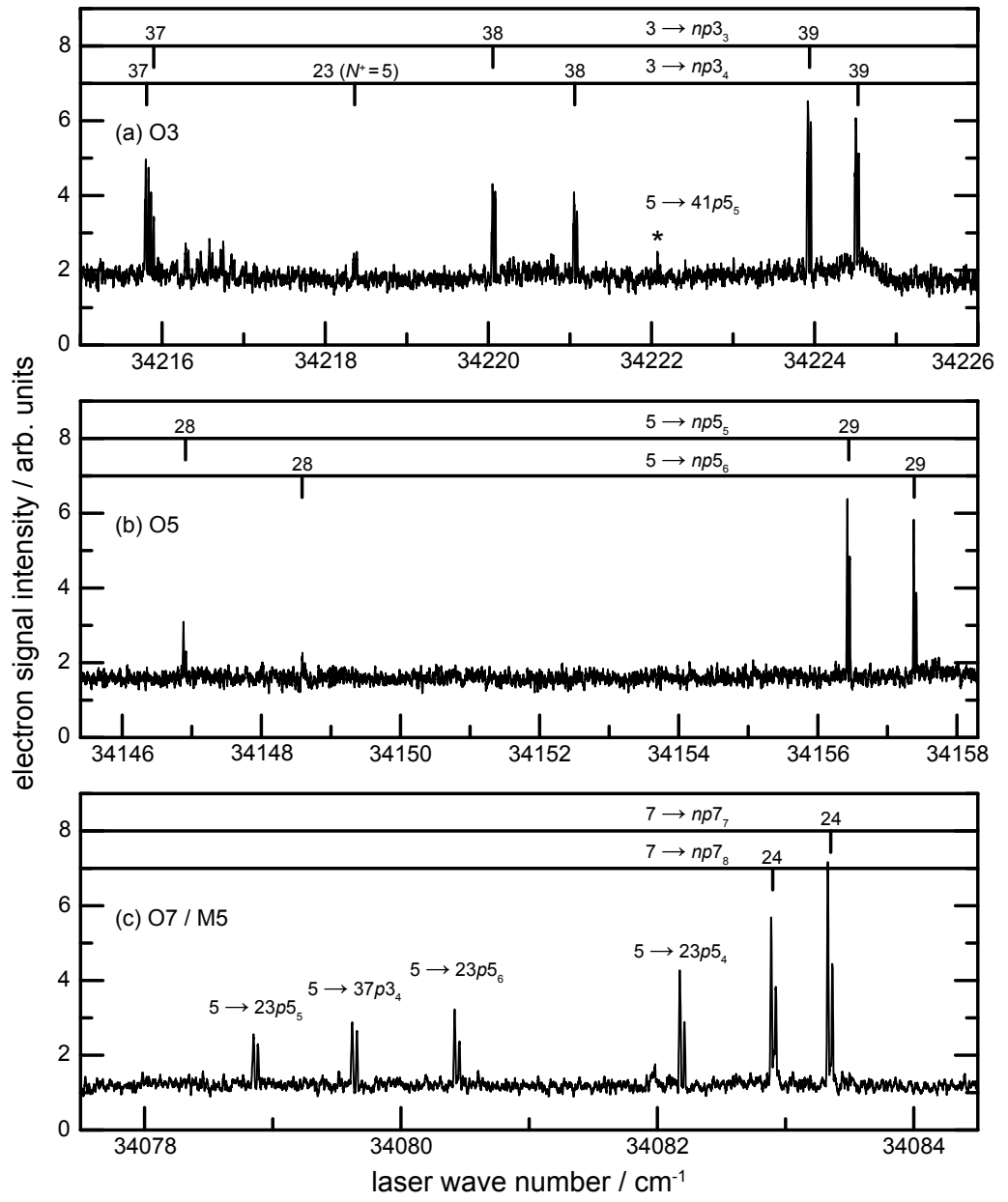

FIG. 5. RSR-TI spectra of ${ }^{4} \mathrm{He}_{2}$ recorded in regions (a) O3, (b) O5, and (c) O7 and M5 (see Fig. 2 for the definition of these regions). The line marked with an asterisk in panel (a) is affected by $p$ - $f$ interaction (see Section III F for discussion).

$n p 1_{N}$ Rydberg states are dominant in region Q1 and resolved in the range $n=95-145$ (see assignment bars in Fig. 4a). Region Q3 is located only $4.9 \mathrm{~cm}^{-1}$ below region Q1 because the rotational constant of $\mathrm{He}_{2}^{+}$is slightly smaller than that of $\mathrm{He}_{2}^{*} \cdot{ }^{18}$ The RSR-TI spectrum recorded in region Q3 is depicted in Fig. 4b and overlaps with regions Q1 and Q5. No regular series of lines converging to the Q3 ionization limit is visible in the spectrum, presumably because the lifetime of the $n p 3_{N}$ Rydberg states around $n=100$ is shorter than $2 \mu \mathrm{s}$. The decay mechanism is rapid rotational autoionization into the $N^{+}=1$ continuum for the $N=2$ levels and rotational autoionization induced by the $p-f$ interaction and by the stray electric field for the $N=3,4$ levels, as explained in Section IIIF. In region Q5, on the other hand, a regular Rydberg series is observed (see Fig. 4c), which can be assigned to the $n p 5_{6}$ Rydberg series on the basis of the calculations presented in Ref. 18. The spectrum recorded in region 


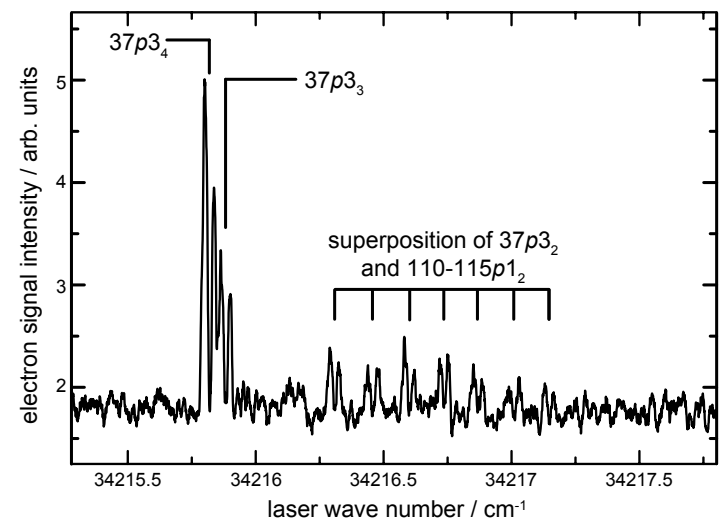

FIG. 6. Enlarged view of the observed RSR-TI spectrum in region O3 near the $3 \rightarrow 37 p 3_{N}$ transitions.

Q7, presented in Fig. 4d, does not reveal any clearly resolved Rydberg series converging to the $N^{+}=7$ ionization threshold. The $n p 7_{N}$ Rydberg states with $n<150$ autoionize before the electric-field pulse is applied and are therefore not detected. For $n>150$, the signal of the unresolved $n p 7_{N}$ Rydberg series gradually increases up $34274.3 \mathrm{~cm}^{-1}$, which corresponds to the position where the $n \approx 400$ Rydberg states are expected. This increase is attributed to the increase of the lifetime with growing $n$ values and is consistent with the fact that the autoionization lifetimes of Rydberg states scale as $n^{4-5}$ in the presence of stray electric fields. ${ }^{43,44}$ Rydberg states with $n>400$ are not stable in the presence of the stray electric field of $\sim 0.01 \mathrm{~V} / \mathrm{cm}$.

The RSR-TI spectra recorded in the regions O3-O7 and M5 are presented in Fig. 5. In these regions, the sharp structures correspond to transitions to Rydberg states that are detected following field-induced rotational autoionization. In the spectrum recorded in region $\mathrm{O} 3$ (see Fig. 5a), two Rydberg series, the $3 \rightarrow n p 3_{3}$ and $3 \rightarrow n p 3_{4}$ series, are visible, as indicated by the assignment bars. Whereas the $n p 3_{3}$ Rydberg series is regular, the members of the $n p 3_{4}$ series between $n=37$ and $n=39$ are strongly perturbed by a rotational channel interaction with the $23 p 5_{4}$ Rydberg state. The rotational channel interaction leads to pronounced level shifts which are quantitatively analyzed in Section III C. Members of two Rydberg series are also observed in the O5 and O7 regions, as illustrated in Fig. 5b and 5c, respectively. The O7 and M5 regions overlap and the four lowest transitions observed in Fig. 5c are assigned to M5 transitions on the basis of their fine structure (see Section III B).

The assignment of the line marked with an asterisk in Fig. 5a is discussed in Section III F. An additional spectral structure, displayed on an enlarged scale in Fig. 6, is observed near 
TABLE II. Term values and fine-structure splittings of the $\left(v^{\prime \prime}=0, N^{\prime \prime}=1-7\right)$ rovibrational levels of the $2 \mathrm{~s} \sigma a^{3} \Sigma_{u}^{+}$metastable state of ${ }^{4} \mathrm{He}_{2}$ taken from Ref. 28. All values are given in units of $\mathrm{cm}^{-1}$.

\begin{tabular}{ccccc}
\hline \hline$N^{\prime \prime}$ & $\tilde{\nu}_{\text {obs }}{ }^{\mathrm{a}}$ & $F_{1}{ }^{\mathrm{b}}$ & $F_{2}{ }^{\mathrm{b}}$ & $F_{3}{ }^{\mathrm{b}}$ \\
\hline 1 & $15.1760(1)$ & 0.0049 & -0.0243 & 0.0491 \\
3 & $90.9889(3)$ & 0.0079 & -0.0243 & 0.0199 \\
5 & $227.1697(8)$ & 0.0089 & -0.0242 & 0.0167 \\
7 & $423.2366(16)$ & 0.0094 & -0.0241 & 0.0155 \\
\hline \hline
\end{tabular}

a Term values relative to the hypothetical $\left(v^{\prime \prime}=0, N^{\prime \prime}=0\right)$ level of the $2 \mathrm{~s} \sigma a^{3} \Sigma_{u}^{+}$state and corresponding to the center of gravity of the three fine-structure components.

${ }^{\mathrm{b}}$ Splittings resulting from the spin fine structure. $F_{1}, F_{2}$, and $F_{3}$ denote the fine-structure components with $J^{\prime \prime}=N^{\prime \prime}+1, J^{\prime \prime}=N^{\prime \prime}$, and $J^{\prime \prime}=N^{\prime \prime}-1$, respectively.

$34217 \mathrm{~cm}^{-1}$ in region O3 of the RSR-TI spectrum. The four strong lines on the left-hand side of Fig. 6 correspond to the $N=3$ and $N=4$ orbital fine-structure components of the $37 p 3_{N}$ Rydberg state. The weaker set of lines located in the center of Fig. 6 appears at the position where the $37 p 3_{2}$ Rydberg state is expected. This state is not observed as a single resonance because of the rotational channel interaction with members of the $n p 1_{2}$ Rydberg series around $n=112$ which gain intensity from the strong $37 p 3_{2}$ transition. The splitting observed for all transitions in Fig. 6 originates from the fine structure of the $N^{\prime \prime}=3$ initial state, as discussed in the next section.

\section{B. Determination of term values}

The term values of the levels reported in this article correspond to their wave numbers relative to the hypothetical $\left(v^{\prime \prime}=0, N^{\prime \prime}=0\right)$ level of the $2 \mathrm{~s} \sigma a^{3} \Sigma_{u}^{+}$metastable state of $\mathrm{He}_{2}$. The term values of the rotational levels of the metastable state are known accurately from high-resolution near-infrared emission spectroscopy ${ }^{28}$ and are listed in the second column of Table II. Each of these rotational levels is split into three components labeled $F_{1}$ (for $\left.J^{\prime \prime}=N^{\prime \prime}+1\right), F_{2}\left(\right.$ for $\left.J^{\prime \prime}=N^{\prime \prime}\right)$, and $F_{3}\left(\right.$ for $\left.J^{\prime \prime}=N^{\prime \prime}-1\right)$ by the spin-spin and spin-rotation interactions. The spin fine structure reported in Ref. 28 is included in Table II.

The term values of triplet $n p$ Rydberg states of $\mathrm{He}_{2}$ were determined from the spectra presented in the previous section by adding the observed transition wave numbers to the term values of the initial metastable states given in Table II. The spin fine structure of the metastable states, which was partially resolved in the measurements (see, e.g., Fig. 3), had 


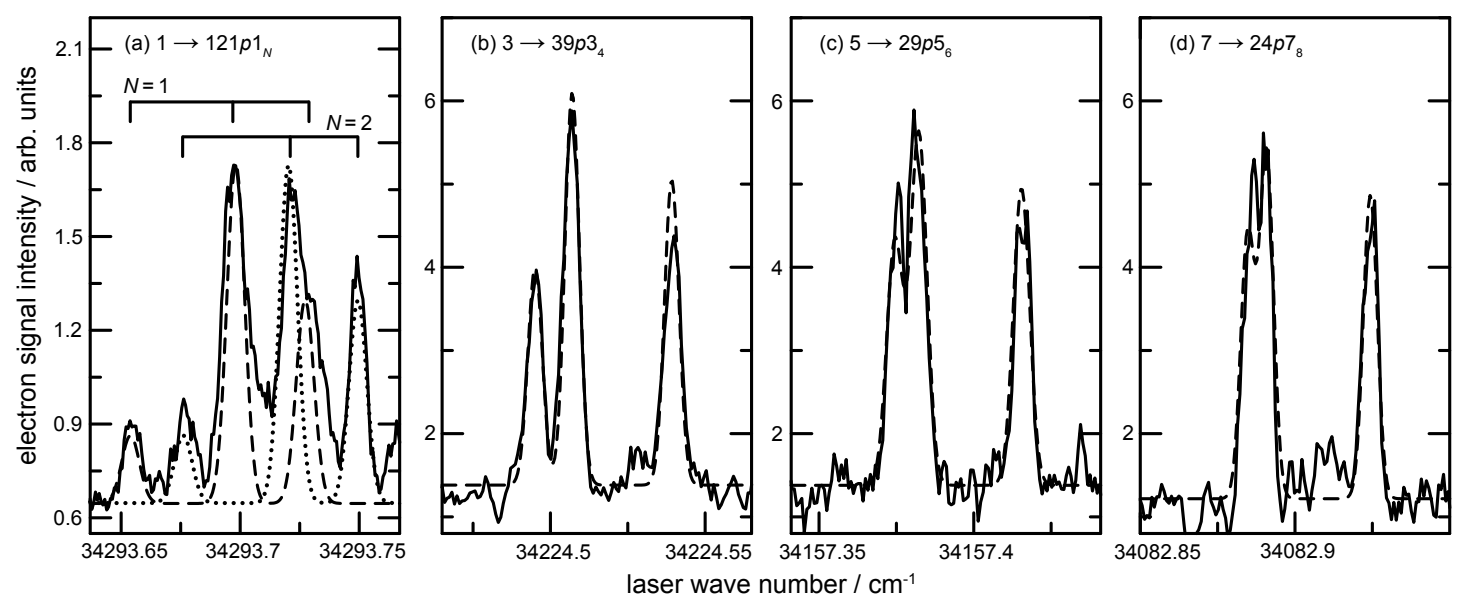

FIG. 7. Enlarged view of the observed RSR-TI spectra around selected transitions (solid lines). The dashed and dotted lines represent the simulated lineshapes (see text for details).

to be taken into account in the lineshape model in order to extract reliable experimental transition wave numbers. The spin fine structure of the final triplet $n p$ Rydberg states, which is expected to scale as $n^{-3}$, could not be resolved and was neglected in the analysis. For each transition, four fit parameters were used to model the observed lineshape: the wave number corresponding to the center of gravity of the transition, the linewidth, a constant background signal, and the intensity of the central $\left(F_{1}\right)$ spin fine-structure component. The intensity ratio between the $F_{1}, F_{2}$, and $F_{3}$ components was fixed to the ratio expected from the degeneracy factor $2 J^{\prime \prime}+1$. Representative fits of the lineshape model to observed intensity distributions of transitions originating from metastable levels with $N^{\prime \prime} \leq 7$ are presented in Fig. 7 . For the $1 \rightarrow n p 1_{N}$ transitions, which were observed only for $n>86$, the $N=1$ and $N=2$ orbital fine-structure components partially overlap and were therefore fitted simultaneously, as illustrated in Fig. 7a for the $1 \rightarrow 121 p 1_{N}$ transitions. The dashed and dotted lines in Fig. 7a represent the simulated contributions of the $N=1$ and $N=2$ orbital fine-structure components, respectively. The $N^{\prime \prime}$ dependence of the fine structure observable in Fig. 7 turned out to be very useful in the analysis because it enabled the unambiguous assignment of the $N^{\prime \prime}$ quantum number. All assignments presented in the previous section were confirmed in this way.

The experimental term values of all triplet $n p$ Rydberg states observed in the RSRTI spectra recorded in regions Q1-Q7 are listed in Table III. The $N=1$ and $N=2$ Rydberg series converging to the $N^{+}=1$ threshold have approximately the same intensity 
TABLE III. Experimental term values of triplet $n p$ Rydberg states of ${ }^{4} \mathrm{He}_{2}$ observed in the RSRTI spectra recorded in the regions Q1-Q7 (see Fig. 4) and comparison with the results of MQDT calculations. All values are given in units of $\mathrm{cm}^{-1}$.

\begin{tabular}{|c|c|c|c|c|c|c|c|c|}
\hline \multirow[b]{2}{*}{$n$} & \multicolumn{2}{|c|}{$n p 1_{1}$ series } & \multicolumn{2}{|c|}{$n p 1_{2}$ series } & \multicolumn{2}{|c|}{$n p 3_{4}$ series } & \multicolumn{2}{|c|}{$n p 5_{6}$ series } \\
\hline & $\tilde{\nu}_{\text {obs }}$ & $\mathrm{O}-\mathrm{c}^{\mathrm{a}}$ & $\tilde{\nu}_{\text {obs }}$ & $\mathrm{O}-\mathrm{c}^{\mathrm{a}}$ & $\tilde{\nu}_{\text {obs }}$ & $\mathrm{O}-\mathrm{c}^{\mathrm{a}}$ & $\tilde{\nu}_{\text {obs }}$ & $\mathrm{O}-\mathrm{c}^{\mathrm{a}}$ \\
\hline 87 & 34301.8616 & -0.0005 & 34301.8776 & 0.0004 & & & & \\
\hline 88 & 34302.1901 & -0.0004 & 34302.1952 & -0.0002 & & & & \\
\hline 89 & 34302.5074 & -0.0004 & 34302.4985 & 0.0001 & & & & \\
\hline 90 & 34302.8145 & -0.0003 & 34302.7814 & -0.0005 & & & & \\
\hline 91 & 34303.1109 & -0.0007 & 34303.0385 & 0.0004 & & & & \\
\hline 92 & 34303.3980 & -0.0007 & 34303.2689 & -0.0010 & & & & \\
\hline 93 & 34303.6763 & -0.0004 & 34303.7537 & 0.0012 & & & & \\
\hline 94 & 34303.9456 & -0.0002 & 34304.0059 & 0.0011 & & & & \\
\hline 95 & 34304.2064 & -0.0000 & 34304.2539 & -0.0011 & 34375.1054 & -0.0012 & & \\
\hline 96 & 34304.4588 & -0.0002 & & & 34375.3437 & -0.0002 & & \\
\hline 97 & 34304.7023 & -0.0014 & 34304.7396 & 0.0001 & 34375.5699 & -0.0011 & & \\
\hline 98 & 34304.9412 & 0.0002 & 34304.9736 & 0.0013 & 34375.7892 & -0.0004 & 34503.3314 & -0.0010 \\
\hline 99 & 34305.1709 & -0.0003 & 34305.1993 & 0.0007 & 34376.0032 & 0.0005 & 34503.5596 & -0.0014 \\
\hline 100 & 34305.3933 & -0.0012 & 34305.4193 & 0.0008 & 34376.2117 & -0.0008 & 34503.7828 & 0.0001 \\
\hline 101 & 34305.6109 & -0.0002 & 34305.6328 & 0.0007 & & & 34503.9974 & -0.0005 \\
\hline 102 & 34305.8213 & -0.0002 & 34305.8383 & -0.0012 & & & 34504.2073 & 0.0005 \\
\hline 103 & 34306.0248 & -0.0009 & 34306.0406 & -0.0003 & & & 34504.4101 & 0.0004 \\
\hline 104 & 34306.2229 & -0.0011 & 34306.2370 & 0.0004 & 34377.2143 & 0.0002 & 34504.6066 & 0.0001 \\
\hline 105 & 34306.4166 & -0.0002 & 34306.4265 & 0.0007 & 34377.4017 & -0.0002 & 34504.7988 & -0.0007 \\
\hline 106 & 34306.6037 & -0.0003 & 34306.6115 & 0.0018 & 34377.5853 & 0.0001 & 34504.9846 & 0.0005 \\
\hline 107 & $34306.7875^{b}$ & 0.0014 & $34306.7875^{b}$ & -0.0003 & 34377.7647 & 0.0009 & 34505.1656 & 0.0006 \\
\hline 108 & $34306.9616^{\mathrm{b}}$ & -0.0015 & $34306.9616^{\mathrm{b}}$ & 0.0017 & 34377.9385 & 0.0007 & 34505.3411 & 0.0003 \\
\hline 109 & 34307.1361 & 0.0006 & 34307.1264 & 0.0002 & 34378.1087 & 0.0013 & 34505.5125 & 0.0007 \\
\hline 110 & 34307.3025 & -0.0003 & 34307.2846 & -0.0005 & & & 34505.6788 & 0.0006 \\
\hline 111 & 34307.4656 & -0.0002 & 34307.4345 & -0.0013 & & & 34505.8412 & 0.0011 \\
\hline 112 & 34307.6241 & -0.0003 & 34307.5770 & -0.0007 & & & 34505.9999 & 0.0023 \\
\hline 113 & 34307.7794 & 0.0006 & 34307.7131 & 0.0001 & & & 34506.1532 & 0.0023 \\
\hline 114 & 34307.9283 & -0.0009 & & & & & 34506.3029 & 0.0026 \\
\hline 115 & 34308.0759 & 0.0002 & 34308.1183 & -0.0005 & & & 34506.4485 & 0.0028 \\
\hline 116 & 34308.2183 & -0.0001 & 34308.2562 & 0.0017 & & & 34506.5899 & 0.0026 \\
\hline 117 & 34308.3576 & 0.0002 & 34308.3889 & 0.0002 & & & 34506.7291 & 0.0038 \\
\hline 118 & 34308.4925 & -0.0005 & 34308.5212 & 0.0007 & & & 34506.8634 & 0.0037 \\
\hline 119 & 34308.6246 & -0.0005 & 34308.6505 & 0.0008 & & & 34506.9954 & 0.0046 \\
\hline 120 & 34308.7541 & 0.0002 & 34308.7775 & 0.0013 & & & 34507.1238 & 0.0053 \\
\hline 121 & 34308.8790 & -0.0006 & 34308.9012 & 0.0014 & & & 34507.2484 & 0.0053 \\
\hline 122 & 34309.0021 & -0.0001 & 34309.0228 & 0.0021 & & & 34507.3698 & 0.0053 \\
\hline 123 & 34309.1232 & 0.0015 & 34309.1405 & 0.0016 & & & 34507.4890 & 0.0062 \\
\hline 124 & 34309.2394 & 0.0009 & 34309.2564 & 0.0021 & & & 34507.6073 & 0.0047 \\
\hline 125 & 34309.3524 & 0.0000 & 34309.3692 & 0.0022 & & & 34507.7217 & 0.0105 \\
\hline 126 & 34309.4647 & 0.0011 & 34309.4794 & 0.0023 & & & & \\
\hline
\end{tabular}

a Difference between observed term values and term values calculated using the eigenquantum defects from Table VI and the adjusted rovibrational energies of ${ }^{4} \mathrm{He}_{2}^{+}$(see Table VIII).

b The $N=1$ and 2 orbital fine-structure components of the $n=107$ and 108 states could not be resolved. 
TABLE IV. Experimental term values of triplet $n p$ Rydberg states of ${ }^{4} \mathrm{He}_{2}$ observed in regions O3-O7 and M5 (see Fig. 5) and comparison with the results of MQDT calculations. All values are given in units of $\mathrm{cm}^{-1}$.

\begin{tabular}{|c|c|c|c|c|c|}
\hline Label & $\tilde{\nu}_{\text {obs }}$ & $\mathrm{O}-\mathrm{c}^{\mathrm{a}}$ & Label & $\tilde{\nu}_{\text {obs }}$ & $\mathrm{O}-\mathrm{c}^{\mathrm{a}}$ \\
\hline \multicolumn{3}{|c|}{ Negative electronic parity } & \multicolumn{3}{|c|}{ Positive electronic parity } \\
\hline \multirow[t]{2}{*}{$37 p 3_{3}$} & \multirow[t]{2}{*}{34306.8625} & \multirow[t]{2}{*}{-0.0039} & $37 p 3_{4}$ & 34306.7999 & 0.0068 \\
\hline & & & $23 p 5_{4}$ & 34309.3555 & -0.0030 \\
\hline $38 p 3_{3}$ & 34311.0502 & -0.0027 & $38 p 3_{4}$ & 34312.0515 & -0.0037 \\
\hline $39 p 3_{3}$ & 34314.9179 & -0.0031 & $39 p 3_{4}$ & 34315.5038 & -0.0024 \\
\hline $23 p 5_{5}$ & 34306.0302 & 0.0031 & $23 p 5_{6}$ & 34307.6006 & 0.0090 \\
\hline $28 p 5_{5}$ & 34374.0618 & 0.0009 & $28 p 5_{6}$ & 34375.7730 & 0.0020 \\
\hline $29 p 5_{5}$ & 34383.6051 & -0.0007 & $29 p 5_{6}$ & 34384.5609 & 0.0019 \\
\hline \multirow[t]{2}{*}{$24 p 7_{7}$} & 34506.5783 & 0.0056 & $24 p 7_{8}$ & 34506.1368 & 0.0082 \\
\hline & lean-square: & 0.0033 & \multicolumn{2}{|c|}{ root-mean-square: } & 0.0054 \\
\hline
\end{tabular}

${ }^{a}$ Difference between observed term values and term values calculated using the eigenquantum defects from Table VI. The rovibrational energy levels and potential-energy curve of ${ }^{4} \mathrm{He}_{2}^{+}$in the $X^{+2} \Sigma_{u}^{+}$ ground state were taken from recent $a b$ initio calculations. ${ }^{7}$

(see Fig. 7a), except near the $96 p 1_{2}$ and $114 p 1_{2}$ Rydberg states, where the intensity of the $N=2$ series is reduced because of rotational channel interactions with members of the $n p 3_{2}$ series. No members of Rydberg series with $N=N^{+}-1$ were observed in regions Q3-Q7, presumably because they are subject to fast rotational autoionization. In regions Q3 and Q5 of the RSR-TI spectrum, only transitions to the $N=N^{+}+1$ orbital fine-structure components were observed and transitions to the $n p 3_{4}$ Rydberg states appear much weaker than transitions to the $n p 5_{6}$ Rydberg states. These observations can be explained by the autoionization lifetimes of the final Rydberg states (see Section III F below).

The term values of $n p$ Rydberg states observed in regions O3-O7 and M5 are listed in Table IV. The $37 p 3_{4}$ and $23 p 5_{4}$ Rydberg states were observed from the $N^{\prime \prime}=3$ and $N^{\prime \prime}=5$ levels of the metastable state (see panels a and c of Fig. 5). The term values determined from these transitions differ by less than $0.004 \mathrm{~cm}^{-1}$, which is within the estimated accuracy of the wave-number calibration (see Table I).

\section{Adjustment of eigenquantum-defect parameters}

To analyze the spectra, we used MQDT as developed by Ch. Jungen and his coworkers. ${ }^{40,45-54}$ MQDT using $\eta$ eigenquantum defects ${ }^{47-49,54}$ was applied to quantitatively describe the ob- 
served term values of triplet $n p$ and $n f$ Rydberg states of $\mathrm{He}_{2}$ converging to the $X^{+2} \Sigma_{u}^{+}$ ground state of $\mathrm{He}_{2}^{+}$. MQDT describes these states in terms of eigenquantum-defect functions $\eta_{\ell \ell^{\prime}}^{(\Lambda)}(R, \varepsilon)$, where $R$ is the internuclear distance and $\varepsilon$ is the energy of the Rydberg electron divided by the Rydberg constant:

$$
\varepsilon=\frac{E-E^{+}}{h c \mathcal{R}_{\mathrm{He}_{2}}} .
$$

In Eq. (2), $E$ is the total energy and $E^{+}$is the energy of the ${ }^{4} \mathrm{He}_{2}^{+}$ion core in the $X^{+2} \Sigma_{u}^{+}$ ground state taken from recent ab initio calculations. ${ }^{7}$ The procedure and the program, developed by Ch. Jungen and his coworkers ${ }^{40,45-54}$, to calculate Rydberg spectra from the eigenquantum-defect functions were the same as used in a recent study of the triplet $n s$ and $n d$ Rydberg states of $\mathrm{H}_{2} \cdot{ }^{55}$ In that study, the eigenquantum-defect parameters of $\mathrm{H}_{2}$ were extracted from available $a b$ initio potential-energy curves ${ }^{56-59}$ and subsequently adjusted to fit experimental data. No highly accurate potential-energy curves are available for the lowest Rydberg states of $\mathrm{He}_{2}$ and eigenquantum-defect parameters had to be obtained directly in a fit to experimental term values of triplet $n p$ and $n f$ Rydberg states. As in Ref. 55, the fitting procedure was divided into two steps. In the first step (fit A), the $\eta_{p p}^{(\Pi)}, \eta_{p f}^{(\Pi)}, \eta_{f f}^{(\Pi)}$, $\eta_{f f}^{(\Delta)}$, and $\eta_{f f}^{(\Phi)}$ eigenquantum defects were determined and only Rydberg states of negative electronic parity were considered, i.e., the $n p^{3} \Pi_{g}^{-}, n f^{3} \Pi_{g}^{-}, n f^{3} \Delta_{g}^{-}$, and $n f^{3} \Phi_{g}^{-}$states when using Hund's-case-(b) notation, or the $n p N_{N}^{+}$and $n f N_{N}^{+}$Rydberg states with even values of $N^{+}-N$ when using Hund's-case-(d) notation. The reason for a separate fit of levels with negative electronic parity is that the positions of these levels do not depend on eigenquantumdefect parameters of $\Sigma$ symmetry. The $\eta_{p p}^{(\Sigma)}, \eta_{p f}^{(\Sigma)}$, and $\eta_{f f}^{(\Sigma)}$ eigenquantum defects were then adjusted in the second step (fit B) to reproduce the observed positions of the energy levels with positive electronic parity.

The eigenquantum-defect functions of $\mathrm{He}_{2}$ were determined by adjusting their values at predefined values of $R$ and $\varepsilon$, i.e., $R=1.8 a_{0}, 2.1 a_{0}, 2.4 a_{0}$ and $\varepsilon=0,-1 / 4^{2},-1 / 3^{2}$ for the $\eta_{p p}^{(\Pi)}$ eigenquantum defects adjusted in fit $\mathrm{A}$. The values $\varepsilon=0,-1 / 4^{2},-1 / 3^{2}$ correspond to states with $n \rightarrow \infty, n=4$, and $n=3$, respectively. The values of $\eta_{p p}^{(\Pi)}(R, \varepsilon)$ at arbitrary values of $(R, \varepsilon)$ were obtained by extrapolation using polynomial functions which are quadratic in $R$ and $\varepsilon$. The $\eta_{p f}^{(\Pi)}, \eta_{f f}^{(\Pi)}, \eta_{f f}^{(\Delta)}$, and $\eta_{f f}^{(\Phi)}$ eigenquantum defects were also adjusted in fit A, but were assumed to be independent of $R$ and linearly dependent on $\varepsilon$. In addition to 
TABLE V. Experimental term values of triplet $n p$ and $n f$ Rydberg states of ${ }^{4} \mathrm{He}_{2}$ with $n=4$ and 5 taken from the literature ${ }^{22,27,29}$ and comparison with the results of MQDT calculations. All values are given in units of $\mathrm{cm}^{-1}$.

\begin{tabular}{|c|c|c|c|c|c|c|c|c|c|c|c|c|c|c|}
\hline \multirow[b]{2}{*}{$N$} & \multicolumn{3}{|c|}{$4 p \pi^{3} \Pi_{g}(v=0)^{22}$} & \multicolumn{4}{|c|}{$4 f\left(v^{+}=0\right)^{27}$} & \multicolumn{3}{|c|}{$5 p \pi^{3} \Pi_{g}(v=0)^{22}$} & \multicolumn{4}{|c|}{$5 f\left(v^{+}=0\right)^{29}$} \\
\hline & $\tilde{\nu}_{\text {obs }}$ & $\mathrm{O}-\mathrm{c}^{\mathrm{a}}$ & $\Delta \tilde{\nu}_{p f}^{\mathrm{b}}$ & $N^{+}$ & $\tilde{\nu}_{\text {obs }}$ & $\mathrm{O}-\mathrm{c}^{\mathrm{a}}$ & $\Delta \tilde{\nu}_{p f}^{\mathrm{b}}$ & $\tilde{\nu}_{\text {obs }}$ & $\mathrm{O}-\mathrm{c}^{\mathrm{a}}$ & $\Delta \tilde{\nu}_{p f}^{\mathrm{b}}$ & $N^{+}$ & $\tilde{\nu}_{\text {obs }}$ & $\mathrm{o}-\mathrm{c}^{\mathrm{a}}$ & $\Delta \tilde{\nu}_{p f}^{\mathrm{b}}$ \\
\hline \multicolumn{15}{|c|}{ Used in fit A (levels with negative electronic parity) } \\
\hline 1 & 27207.12 & -0.04 & -0.14 & 3 & 27492.24 & 0.01 & 0.12 & 29799.60 & 0.02 & -0.08 & 3 & 29978.33 & 0.07 & 0.07 \\
\hline \multirow[t]{3}{*}{3} & 27278.36 & -0.02 & -0.16 & 1 & 27462.42 & 0.07 & 0.04 & 29870.63 & -0.01 & -0.11 & 1 & 29931.15 & 0.08 & 0.03 \\
\hline & & & & 3 & 27529.58 & -0.01 & 0.04 & & & & 3 & 29995.96 & 0.10 & 0.04 \\
\hline & & & & 5 & 27632.97 & 0.01 & 0.05 & & & & & & & \\
\hline \multirow[t]{3}{*}{5} & 27406.29 & -0.03 & -0.18 & 3 & 27526.41 & 0.05 & 0.08 & 29999.56 & -0.00 & 1.12 & 3 & 29996.03 & ${ }^{c}-0.02$ & -1.19 \\
\hline & & & & 5 & 27659.77 & -0.01 & 0.05 & & & & 5 & 30125.96 & 0.16 & 0.04 \\
\hline & & & & 7 & 27820.57 & -0.04 & 0.04 & & & & 7 & 30297.68 & -0.14 & 0.02 \\
\hline \multirow[t]{2}{*}{7} & 27590.42 & -0.06 & -0.26 & 5 & 27651.25 & 0.02 & 0.16 & 30182.21 & -0.08 & -0.01 & 5 & 30122.91 & 0.05 & -0.05 \\
\hline & & & & & & & & & & & 7 & 30309.60 & -0.30 & 0.04 \\
\hline 9 & 27828.41 & 0.00 & -2.21 & 7 & 27835.08 & -0.14 & 2.11 & 30421.57 & -0.07 & -0.03 & 7 & 30305.39 & -0.03 & -0.03 \\
\hline \multicolumn{15}{|c|}{ Used in fit B (levels with positive electronic parity) } \\
\hline \multirow[t]{3}{*}{2} & 27234.64 & -0.04 & -0.17 & 1 & 27424.21 & 0.12 & 0.07 & 29826.56 & 0.05 & -0.12 & 1 & 29909.44 & -0.12 & 0.08 \\
\hline & & & & 3 & 27508.53 & 0.14 & -0.02 & & & & 3 & 29986.43 & -0.24 & -0.01 \\
\hline & & & & 5 & 27617.03 & -0.07 & -0.01 & & & & 5 & 30104.17 & -0.32 & -0.01 \\
\hline \multirow[t]{4}{*}{4} & 27332.03 & 0.03 & -0.25 & 1 & 27431.53 & 0.08 & 0.17 & 29923.38 & 0.02 & 0.68 & 1 & 29913.30 & -0.05 & -0.72 \\
\hline & & & & 3 & 27536.04 & 0.00 & 0.00 & & & & 3 & 30000.85 & 0.04 & 0.01 \\
\hline & & & & 5 & 27648.75 & 0.05 & -0.02 & & & & 5 & 30120.83 & 0.72 & -0.01 \\
\hline & & & & 7 & 27802.71 & -0.23 & -0.01 & & & & & & & \\
\hline \multirow[t]{3}{*}{6} & 27484.00 & 0.29 & -1.25 & 3 & 27501.15 & -0.15 & 1.18 & 30073.53 & 0.05 & 0.04 & 3 & 29983.01 & -0.17 & -0.09 \\
\hline & & & & 5 & 27661.49 & 0.02 & 0.01 & & & & 5 & 30127.76 & 0.15 & 0.03 \\
\hline & & & & & & & & & & & 7 & 30305.85 & 0.49 & -0.01 \\
\hline \multirow[t]{2}{*}{8} & 27692.74 & 0.02 & 0.25 & 5 & 27626.23 & 0.01 & -0.32 & 30278.68 & 0.08 & 0.00 & 5 & 30109.67 & -0.22 & -0.05 \\
\hline & & & & & & & & & & & 7 & 30310.89 & 0.26 & 0.04 \\
\hline
\end{tabular}

${ }^{\text {a }}$ Difference between observed term values and term values calculated using the eigenquantum defects from Table VI including the $p-f$ interaction.

b Calculated shifts induced by the $p-f$ interaction.

${ }^{c}$ The term value of the $5 f 3_{5}\left(v^{+}=0\right)$ Rydberg state was taken from Ref. 22 .

these $9+4 \times 2=17$ fit parameters for the eigenquantum-defect functions, the position of the $N^{+}=1$ ionization threshold relative to the hypothetical $\left(v^{\prime \prime}=0, N^{\prime \prime}=0\right)$ level of the $2 s \sigma a^{3} \Sigma_{u}^{+}$state of $\mathrm{He}_{2}$ was also adjusted during fit A, resulting in a total of 18 adjustable parameters.

In the subsequent fit (fit B), the final values of all 18 fit parameters determined in fit A were used without further adjustments and only the eigenquantum defects of $\Sigma$ symmetry were adjusted. The 12 fit parameters used for the $\eta_{p p}^{(\Sigma)}$ eigenquantum defect are the values 
at $R=1.8 a_{0}, 2.1 a_{0}, 2.4 a_{0}, 2.7 a_{0}$ and $\varepsilon=0,-1 / 4^{2},-1 / 3^{2}$. The values of $\eta_{p p}^{(\Sigma)}(R, \varepsilon)$ at arbitrary values of $(R, \varepsilon)$ were obtained by extrapolation using polynomial functions which are cubic in $R$ and quadratic in $\varepsilon$. The $\eta_{f f}^{(\Sigma)}$ eigenquantum defect was assumed to be independent of $R$ and linearly dependent on $\varepsilon$, and the $\eta_{p f}^{(\Sigma)}$ eigenquantum defect was assumed to be independent of $R$ and $\varepsilon$. The total number of fit parameters adjusted in fit $\mathrm{B}$ was therefore $12+2+1=15$.

The experimental data set used in fit A consisted of 261 triplet $n p$ and $n f$ Rydberg states with negative electronic parity, $v^{+} \leq 2, N^{+} \leq 19$, and $n$ in the range 2-39. In fit B, 230 triplet $n p$ and $n f$ Rydberg states with positive electronic parity, $v^{+} \leq 2, N^{+} \leq 9, N \leq 8$, and $n$ in the range 2-39 were used. From the total of 491 energy levels, 15 triplet $n p$ levels were taken from Table IV above, 437 triplet $n p$ levels were taken from measurements carried out by Ginter and coworkers, ${ }^{22-26} 19$ triplet $4 f$ levels were taken from Herzberg and Jungen, ${ }^{27}$ and 20 triplet $5 f$ levels were taken from Kawaguchi et al. ${ }^{29}$ All term values of triplet $n p$ Rydberg states taken from the literature are provided in the supplementary material ${ }^{60}$ and the term values of the $4 f$ and $5 f$ Rydberg states ${ }^{27,29}$ are listed in Table $\mathrm{V}$ together with data on $4 p \pi^{3} \Pi_{g}$ and $5 p \pi^{3} \Pi_{g}$ Rydberg states. ${ }^{22}$ The data extracted from the RSR-TI spectra recorded in regions Q1-Q7 (Table III) were not used, because high- $n$ Rydberg states with $n>86$ are not sensitive to adjustments of eigenquantum defects. These states, however, are sensitive to adjustments of the ionization energies and were used to extract rotational energy spacings in ${ }^{4} \mathrm{He}_{2}^{+}$(see Section III E below). All experimental data were weighted according to their uncertainty, which was assumed to be $0.006 \mathrm{~cm}^{-1}$ for the term values taken from Table IV and $0.1 \mathrm{~cm}^{-1}$ for all term values taken from the literature. The basis used in the MQDT calculations included $n p$ channels up to $v_{\max }^{+}=7$ and $n f$ channels up to $v_{\max }^{+}=3$. An increase of the basis size did not lead to a change of the calculated term values, as was tested using the final eigenquantum-defect parameters.

The final values of all eigenquantum-defect parameters adjusted in fits A and B are given in Table VI and the final term value of the $N^{+}=1$ ionization threshold with respect to the Pauli-forbidden $\left(v^{\prime \prime}=0, N^{\prime \prime}=0\right)$ level of the $2 s \sigma a^{3} \Sigma_{u}^{+}$state was determined to be $34316.391 \mathrm{~cm}^{-1}$ (standard deviation $0.002 \mathrm{~cm}^{-1}$, absolute uncertainty $0.010 \mathrm{~cm}^{-1}$ ). The differences between the observed and calculated term values are listed next to the experimental term values in Table IV, Table V, and in the supplementary material. ${ }^{60}$ The MQDT calculations reproduce the 15 triplet $n p$ Rydberg states given in Table IV with a root-mean-square 
TABLE VI. Final values of the eigenquantum-defect parameters $\eta_{\ell \ell^{\prime}}^{(\Lambda)}(R, \varepsilon)$ of $\mathrm{He}_{2}$. The standard deviation in units of the last digit is given in parentheses.

\begin{tabular}{lccccc}
\hline \hline$\ell \ell^{\prime}$ & $\Lambda$ & $R / a_{0}$ & $\varepsilon=0$ & $\varepsilon=-1 / 4^{2}$ & $\varepsilon=-1 / 3^{2}$ \\
\hline & $\Pi$ & From fit A & to levels with negative electronic parity) & \\
$p p$ & $\Pi$ & 1.8 & $0.08698(18)$ & $0.09236(7)$ & $0.09719(5)$ \\
$p p$ & $\Pi$ & 2.1 & $0.06962(3)$ & $0.07385(1)$ & $0.07776(1)$ \\
$p p$ & $\Pi$ & 2.4 & $0.05463(10)$ & $0.05794(4)$ & $0.06125(3)$ \\
$p f$ & $\Pi$ & $2.1^{\mathrm{a}}$ & $0.00272(21)$ & $0.00176(4)$ & \\
$f f$ & $\Pi$ & $2.1^{\mathrm{a}}$ & $0.01232(16)$ & $0.01075(3)$ & \\
$f f$ & $\Delta$ & $2.1^{\mathrm{a}}$ & $0.00409(20)$ & $0.00339(3)$ & \\
$f f$ & $\Phi$ & $2.1^{\mathrm{a}}$ & $-0.00762(20)$ & $-0.00858(4)$ & \\
& & From fit B (to levels with positive electronic parity) & \\
$p p$ & $\Sigma$ & 1.8 & $-0.2405(14)$ & $-0.2341(5)$ & $-0.2294(8)$ \\
$p p$ & $\Sigma$ & 2.1 & $-0.2240(2)$ & $-0.2182(1)$ & $-0.2128(1)$ \\
$p p$ & $\Sigma$ & 2.4 & $-0.1973(7)$ & $-0.1943(25)$ & $-0.1894(4)$ \\
$p p$ & $\Sigma$ & 2.7 & $-0.1332(19)$ & $-0.1472(52)$ & $-0.1499(8)$ \\
$p f$ & $\Sigma$ & $2.1^{\mathrm{a}}$ & $0.0022(3)^{\mathrm{b}}$ & $0.0022(3)^{\mathrm{b}}$ & \\
$f f$ & $\Sigma$ & $2.1^{\mathrm{a}}$ & $0.0140(4)$ & $0.01324(4)$ & \\
\hline \hline
\end{tabular}

a All $p f$ and $f f$ eigenquantum defects were assumed to be $R$ independent.

b The $\eta_{p f}^{(\Sigma)}$ eigenquantum defects were assumed to be energy independent.

(rms) deviation of $0.0045 \mathrm{~cm}^{-1}$. For the low- $n p$ and $n f$ Rydberg states, the rms deviation amounts to $0.103 \mathrm{~cm}^{-1}$ for the levels with negative electronic parity and $0.197 \mathrm{~cm}^{-1}$ for the levels with positive electronic parity. Several levels of the $4 p \pi^{3} \Pi_{g}$ and $5 p \pi^{3} \Pi_{g}$ states are strongly perturbed by close-lying $4 f$ and $5 f$ Rydberg states via the $p$ - $f$ interaction, as was pointed out previously. ${ }^{22,27,29}$ Our MQDT calculations accurately reproduce these perturbations as can be seen in Table $\mathrm{V}$. The calculated shifts induced by the $p-f$ interaction are also included in Table $\mathrm{V}$ and can be as large as $2.2 \mathrm{~cm}^{-1}$.

Because the quantum defect of the $n p \sigma^{3} \Sigma_{g}^{+}$states $(\sim-0.2)$ is very different from the quantum defect of the $n f$ states $(\sim 0.0)$, the corresponding series are well separated. The $p-f$ interaction does not induce significant shifts in low- $n$ Rydberg states of $\Sigma$ symmetry. The $\eta_{p f}^{(\Sigma)}$ eigenquantum-defect parameter is therefore poorly defined by the data set and we expect the uncertainty of its value listed in Table VI to be larger than the standard deviation.

The final triplet $\eta_{p p}^{(\Sigma)}(R, \varepsilon)$ and $\eta_{p p}^{(\Pi)}(R, \varepsilon)$ eigenquantum-defect functions of $\mathrm{He}_{2}$ are displayed in Fig. 8. Their values at $R=2.1 a_{0}$ and $\varepsilon=0$ are $-0.2240(2)$ and $0.06962(3)$, respectively. Whereas the value of the $\eta_{p p}^{(\Pi)}$ eigenquantum defect lies between the values 


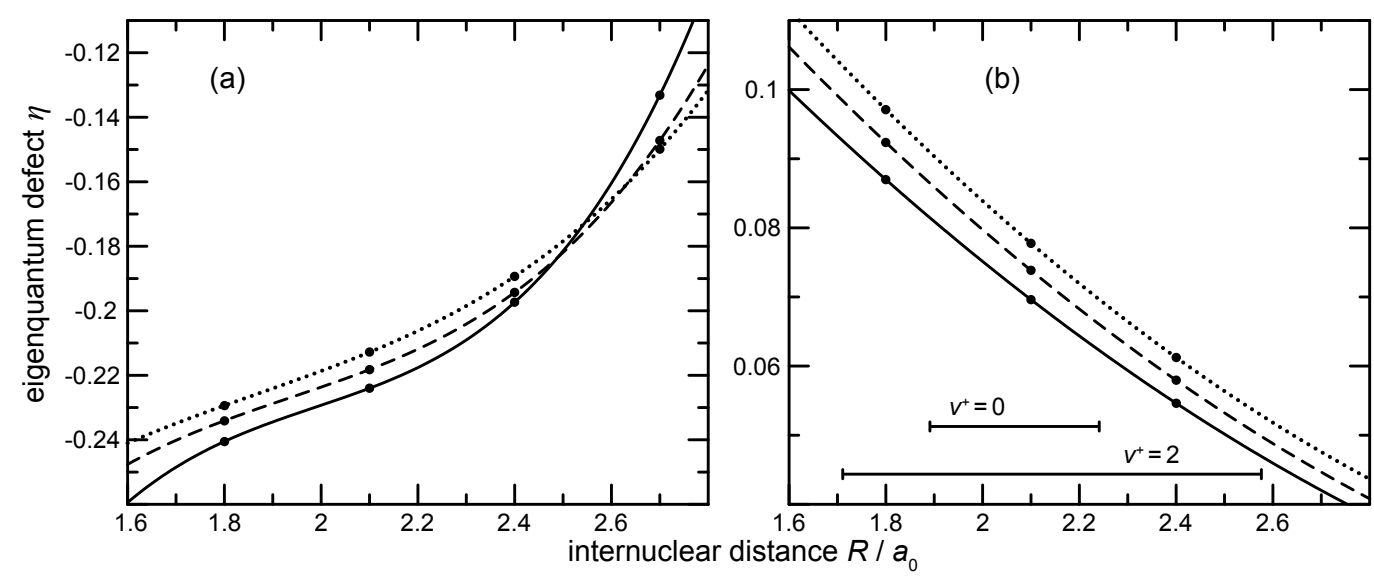

FIG. 8. (a) $\eta_{p p}^{(\Sigma)}(R, \varepsilon)$ and (b) $\eta_{p p}^{(\Pi)}(R, \varepsilon)$ eigenquantum-defect functions of $\mathrm{He}_{2}$ after adjustment to experimental data at $\varepsilon=0$ (solid lines), $\varepsilon=-1 / 4^{2}$ (dashed lines) and $\varepsilon=-1 / 3^{2}$ (dotted lines). The dots represent the fit parameters adjusted in fits A and B (see Table VI). The intervals given in panel (b) depict the classically allowed range of $R$ values for $\mathrm{He}_{2}^{+}$in the states $\left(v^{+}=0, N^{+}=1\right)$ and $\left(v^{+}=2, N^{+}=1\right)$.

determined in Ref. 18 for the $v^{+}=0$ and $v^{+}=1$ Rydberg series, our $\eta_{p p}^{(\Sigma)}$ eigenquantum defect is lower by 1 than in Ref. 18. A shift by 1 does not change the resulting level energies, but changes the calculated rotational $\left(N^{+}\right)$character in the vicinity of rotational channel interactions. Such an interaction is encountered, e.g., in the $n p 3_{4}$ Rydberg series around $n=38$, as already discussed in Section III A in the context of Fig. 5 (see lower assignment bar in panel a). Using our $p p$ eigenquantum defects, the second line assigned to the $N^{\prime \prime} \rightarrow N=4$ series and observed at $34218.4 \mathrm{~cm}^{-1}$ in Fig. 5a is calculated to have the largest $N^{+}=5$ character $(73 \%)$, which explains its weak intensity. When shifting the $\eta_{p p}^{(\Sigma)}$ eigenquantum defect by 1 , the same member is calculated to have only $28 \% N^{+}=5$ character and $72 \% N^{+}=3$ character, whereas the first member of the series observed at $34215.8 \mathrm{~cm}^{-1}$ in Fig. 5a (labeled $37 p 3_{4}$ ) is calculated to have $75 \% N^{+}=5$ character, which agrees less well with the observed intensity distribution.

\section{Determination of potential-energy curves of low-np Rydberg states of $\mathrm{He}_{2}$}

Potential-energy curves and eigenquantum-defect functions are closely related and can be transformed into each other. ${ }^{45,47,48,50,53-55}$ The adjusted $\eta_{p p}^{(\Lambda)}(R, \varepsilon)$ eigenquantum-defect functions derived in the previous section were used to determine the potential-energy curves of the $n p \sigma^{3} \Sigma_{g}^{+}$and $n p \pi^{3} \Pi_{g}$ Rydberg states of $\mathrm{He}_{2}$ with $n \leq 5$ in the range of $R=1.7-2.7 a_{0}$. 
TABLE VII. Born-Oppenheimer potential-energy curves $E_{\mathrm{BO}}$ of the lowest four $n p \sigma^{3} \Sigma_{g}^{+}$and $n p \pi^{3} \Pi_{g}$ Rydberg states of $\mathrm{He}_{2}$ (in units of the Hartree energy $E_{\mathrm{h}}$ ). The adiabatic corrections $E_{\text {ad }} / h c$ are given in units of $\mathrm{cm}^{-1}$.

\begin{tabular}{|c|c|c|c|c|c|c|c|c|}
\hline$R / a_{0}$ & $E_{\mathrm{BO}} / E_{\mathrm{h}}$ & $E_{\text {ad }} / h c$ & $E_{\mathrm{BO}} / E_{\mathrm{h}}$ & $E_{\text {ad }} / h c$ & $E_{\mathrm{BO}} / E_{\mathrm{h}}$ & $E_{\text {ad }} / h c$ & $E_{\mathrm{BO}} / E_{\mathrm{h}}$ & $E_{\mathrm{ad}} / h c$ \\
\hline & $2 p \sigma c^{3} \Sigma$ & & $3 p \sigma^{3} \Sigma_{g}^{+}$ & & $4 p c$ & & $5 p \sigma^{3} \Sigma_{g}^{+}$ & \\
\hline 1.7 & -5.07995477 & 155.89 & $408949^{g}$ & 155.05 & 379 & 154.74 & $10549^{9}$ & 154.60 \\
\hline 1.8 & 9099084 & 154.28 & -5.03464699 & 153.44 & 426889 & 153.13 & 454093 & 152.98 \\
\hline 1.9 & 743767 & 153.06 & 1060425 & 152.20 & 013898 & .89 & 37865 & 1.75 \\
\hline 2.0 & 048191 & 152.13 & -5.04313911 & 151.26 & 2258203 & 150.95 & 278966 & 150.81 \\
\hline 2.1 & & 15 & 316926 & & -5 & & 68491 & .10 \\
\hline 2.2 & -5.09986975 & 150.93 & 1140876 & 150.05 & 2063 & 149.74 & 077181 & 149.59 \\
\hline 2.3 & & 150.59 & & & & & 300 & .24 \\
\hline 2.4 & 661 & 150.37 & 3460 & 149.47 & -5 & 14 & 565 & 149.00 \\
\hline 2.5 & & 150.25 & & & & & 45 & .87 \\
\hline 2.6 & 3726460 & 150.22 & -5.02581421 & 149.29 & -5 . & 148.97 & 279 & 148.82 \\
\hline 2.7 & -5.08372937 & 150.25 & -5.02126928 & 149.31 & 963117 & 148.98 & 459 & 148.83 \\
\hline & \multicolumn{2}{|c|}{$2 p \pi b^{3} \Pi_{g}$} & \multicolumn{2}{|c|}{$3 p \pi^{3} \Pi_{g}$} & \multicolumn{2}{|c|}{$4 p \pi^{3} \Pi_{g}$} & \multicolumn{2}{|l|}{$5 p \pi^{3} \Pi_{g}$} \\
\hline 1.7 & 332824 & 39 & & 21 & -5 & .81 & 664602 & .63 \\
\hline 1.8 & -5 . & 15 & -5 & .59 & -5 & .20 & 68 & .02 \\
\hline 1.9 & -5.127 & 153.51 & -5 & 152.35 & -5 . & 151.96 & 272841 & 151.78 \\
\hline 2.0 & -5.129 & 152.56 & -5.05288 & 151.41 & -5 . & 151.02 & 11 & 0.84 \\
\hline 2.1 & -5.1285 & 151.85 & -5.0525 & 150.70 & -5.02666 & 150.31 & 1486662 & 150.14 \\
\hline 2.2 & -5.125 & 151.32 & -5.05035 & 150.19 & -5 & 149.80 & 1286521 & 149.62 \\
\hline 2.3 & -5.1220 & 150.96 & -5.046928 & 149.83 & 2130 & 149.44 & 0959338 & 149.27 \\
\hline 2.4 & -5.1 & 150.71 & -5 . & 149.59 & -5 & 149.21 & -5 & 149.03 \\
\hline 2.5 & -5.1122 & 150.57 & -5.037866 & 149.46 & -5 & 149.07 & -5.00080779 & 148.90 \\
\hline 2.6 & -5.106 & 150 & -5 & 149.40 & -5 & 149 & 585355 & 148.84 \\
\hline 2.7 & $\mid-5.10135333$ & 150.51 & -5.02760692 & 149.40 & $\mid-5.00236213$ & 149.02 & -4.99078589 & 148.85 \\
\hline
\end{tabular}

The $p$ - $f$ interaction was neglected and the one-channel quantization condition ${ }^{47}$

$$
\frac{\tan \pi \sqrt{-1 / \varepsilon}}{A_{\ell=1}(\varepsilon)}+\tan \pi \eta_{p p}^{(\Lambda)}(R, \varepsilon)=0
$$

was solved for $\varepsilon$ at fixed values of $R$. In Eq. (3), $A_{\ell=1}(\varepsilon)=1-\varepsilon^{2}$ is Ham's scaling factor. ${ }^{48}$ From the solutions $\varepsilon(R)$ of Eq. (3), the Born-Oppenheimer energies $E_{\mathrm{BO}}$ were obtained using

$$
E_{\mathrm{BO}}(R)=\varepsilon(R) \times h c \mathcal{R}_{\infty}+E_{\mathrm{BO}}^{+}(R),
$$


where $\mathcal{R}_{\infty}$ is the Rydberg constant for infinite mass and $E_{\mathrm{BO}}^{+}(R)$ is the Born-Oppenheimer potential-energy curve of the $X^{+2} \Sigma_{u}^{+}$ground state of $\mathrm{He}_{2}^{+}$. In Table VII, the resulting values of $E_{\mathrm{BO}}(R)$ are listed for the lowest four $n p \sigma^{3} \Sigma_{g}^{+}$and $n p \pi^{3} \Pi_{g}$ Rydberg states of $\mathrm{He}_{2}$ using the values of $E_{\mathrm{BO}}^{+}(R)$ from Ref. 7 .

The adiabatic corrections to the Born-Oppenheimer potential-energy curves were estimated from the solutions $\varepsilon(R)$ of Eq. (3) using

$$
E_{\text {ad }}(R)=\varepsilon(R) \times h c\left(\mathcal{R}_{\mathrm{He}_{2}}-\mathcal{R}_{\infty}\right)+E_{\text {ad }}^{+}(R) .
$$

The first term in Eq. (5) is the adiabatic correction originating from the Rydberg electron and $E_{\text {ad }}^{+}(R)$ represent the adiabatic correction to the Born-Oppenheimer potential-energy curve of the $X^{+2} \Sigma_{u}^{+}$ground state of ${ }^{4} \mathrm{He}_{2}^{+}$taken from the supplementary material presented in Ref. 7. The values of $E_{\text {ad }}(R)$ are given in Table VII. The contributions to the adiabatic correction from the Rydberg electron are listed in the supplementary material ${ }^{60}$ and only represent a small fraction of the overall adiabatic correction.

The effect of the $p-f$ interaction on the Born-Oppenheimer potential-energy curves was investigated by solving the corresponding two-channel eigenvalue equation. The potentialenergy curves of $n p$ and $n f$ states do not cross and the shift of the electronic energies induced by the $p-f$ interaction was found to be less than $0.1 \mathrm{~cm}^{-1}$, i.e., smaller than the expected accuracy of the potential-energy curves presented in Table VII.

\section{E. Determination of rotational term values in the $X^{+2} \Sigma_{u}^{+}\left(v^{+}=0\right)$ ground state of ${ }^{4} \mathrm{He}_{2}^{+}$}

The eigenquantum defects determined in Section III C are accurate to better than 0.002 (see Table VI), which allows the prediction of electron binding energies of high- $n$ Rydberg states around $n=100$ with an estimated accuracy of better than $0.0005 \mathrm{~cm}^{-1}$. MQDT can therefore be used to extrapolate the energies of the Rydberg states observed in regions Q1Q5 of the RSR-TI spectra to their limits with high precision. The extrapolation was carried out simultaneously for all observed Rydberg series in a global fitting procedure (referred to as fit $\mathrm{C}$ hereafter). The three fit parameters adjusted in fit $\mathrm{C}$ were the term value of the $\left(v^{+}=0, N^{+}=1\right)$ ionization threshold and the positions of the $N^{+}=3$ and $N^{+}=5$ 


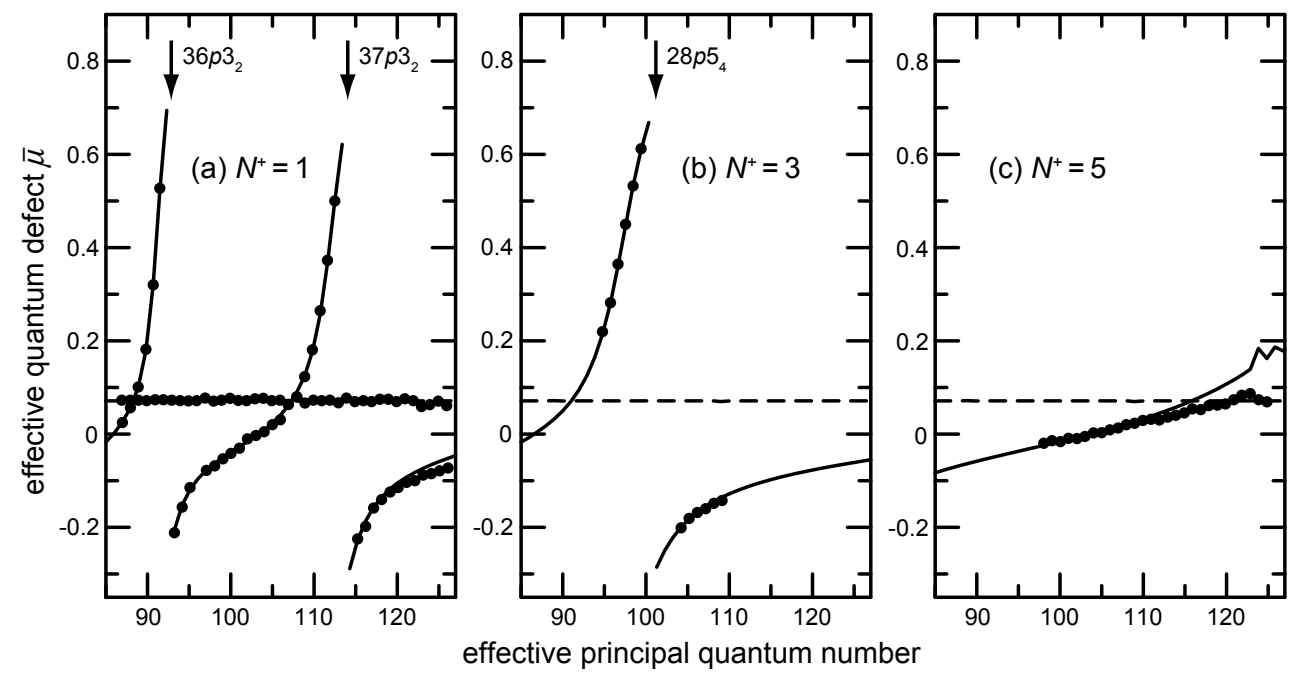

FIG. 9. Experimental (dots) and calculated (lines) quantum-defect plots of triplet $n p$ Rydberg states converging to the (a) $N^{+}=1$, (b) $N^{+}=3$, and (c) $N^{+}=5$ ionization thresholds of ${ }^{4} \mathrm{He}_{2}^{+}$in the $X^{+2} \Sigma_{u}^{+}\left(v^{+}=0\right)$ ground state. Solid and dashed lines depict the calculated effective quantum defects for the Rydberg series with $N=N^{+}+1$ and $N=N^{+}$, respectively.

levels of ${ }^{4} \mathrm{He}_{2}^{+}$in the $X^{+2} \Sigma_{u}^{+}\left(v^{+}=0\right)$ ground state relative to the $N^{+}=1$ level. Further rovibrational energies of ${ }^{4} \mathrm{He}_{2}^{+}$used in the calculations were held fixed at their ab initio values from Ref. 7 .

The experimental data set used in fit $\mathrm{C}$ consisted of 72 triplet $n p$ Rydberg states with $n \leq 110$ listed in Table III. Rydberg states with $n>110$ were not included because their positions are more susceptible to stray electric fields. The same weight was given to all levels used in fit $\mathrm{C}$.

The positions of the $N^{+}=3$ and $N^{+}=5$ rotational levels of ${ }^{4} \mathrm{He}_{2}^{+}$in the $X^{+2} \Sigma_{u}^{+}\left(v^{+}=0\right)$ ground state relative to the $N^{+}=1$ level resulting from fit $\mathrm{C}$ are $70.9372 \mathrm{~cm}^{-1}$ (standard deviation $0.0003 \mathrm{~cm}^{-1}$, absolute uncertainty $0.003 \mathrm{~cm}^{-1}$ ) and $198.3691 \mathrm{~cm}^{-1}$ (standard deviation $0.0003 \mathrm{~cm}^{-1}$, absolute uncertainty $0.006 \mathrm{~cm}^{-1}$ ), respectively. The term value of the $\left(v^{+}=0, N^{+}=1\right)$ ionization threshold was $34316.3831 \mathrm{~cm}^{-1}$ (standard deviation $0.0001 \mathrm{~cm}^{-1}$, absolute uncertainty $\left.0.010 \mathrm{~cm}^{-1}\right)$. The difference between observed and calculated term values for all Rydberg states observed in regions Q1-Q5 of the RSR-TI spectra are included in Table III. The rms deviation of all $n \leq 110$ Rydberg states used in fit $\mathrm{C}$ is $0.0008 \mathrm{~cm}^{-1}$.

The observed and calculated positions of the high- $n$ Rydberg states listed in Table III were transformed into effective quantum defects $\bar{\mu}$ using the Rydberg formula [Eq. (1)] and 
are depicted in Fig. 9 as dots and lines, respectively. Rydberg series with $N=N^{+}$are not affected by rotational channel interactions and their effective quantum defect is constant (see dashed lines in Fig. 9). Strong perturbations are visible for Rydberg series with $N=N^{+}+1$ at the positions of Rydberg states belonging to series converging to the $\mathrm{N}^{+}+2$ ionization thresholds (indicated by vertical arrows). MQDT reproduces these perturbations within the experimental accuracy. Significant deviations are only present at large $n$ values $(n>120$ in Fig. 9a and $n>110$ in Fig. 9c), presumably because of dc Stark shifts induced by the stray electric field.

\section{F. Autoionization dynamics}

In the absence of electric fields and when spins are ignored, the angular-momentum quantum number $N$ of the Rydberg states of $\mathrm{He}_{2}$ investigated here is conserved. For the $n p N_{N=N^{+-1}}^{+}$Rydberg states that are located energetically above the ionization thresholds associated with the $N^{+}-2$ ionic levels, rotational autoionization takes place on the sub-ns time scale up to $n$ values beyond 100. These states are difficult to observe in the PI spectra because their natural linewidths are much larger than the laser linewidth, even at $n=$ 100. They are typically not observed in the RSR-TI spectra either, because autoionization is complete long before the application of the delayed pulsed electric field used for field ionization and electron extraction. Members of the $n p N_{N=N^{+-1}}^{+}$Rydberg series located immediately below the $N^{+}-2$ ionization thresholds, however, are readily observed through their interactions with the long-lived high $n p N^{+}-2_{N=N^{+}-1}$ Rydberg states (see, for example, Fig. 6).

The $n p N_{N=N^{+}}^{+}$and the $n p N_{N=N^{+}+1}^{+}$Rydberg states cannot autoionize to $p$ continua associated with the $N^{+}-2$ ionic levels, but can autoionize to $\ell=3$ continua above the $N^{+}-2$ ionization threshold, the autoionization being mediated by the $p$ - $f$ interaction. The corresponding decay processes are slower than for the $n p N_{N=N^{+-1}}^{+}$Rydberg states, but still, in general, too fast for the corresponding series to be observed by delayed $(2 \mu \mathrm{s})$ pulsed field ionization. Fig. 4 d, for instance, indicates that the lifetimes of the $n p 7_{7,8}$ Rydberg states are less than $1 \mu \mathrm{s}$ up to $n=100$. The Doppler-limited linewidth of the $24 p 7_{7,8}$ resonances in Fig. 5 suggests, however, that their lifetimes is at least about 1 ns at $n=25$ and thus at least about 100 ns at $n=100$ because of the $n^{3}$ scaling of the lifetimes. 


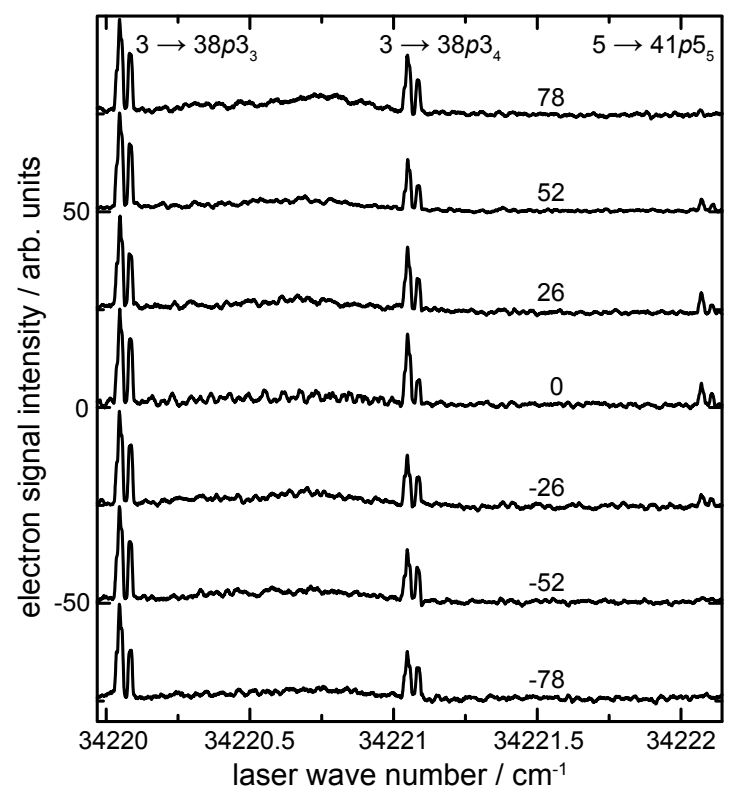

FIG. 10. RSR-TI spectra of ${ }^{4} \mathrm{He}_{2}$ recorded in the presence of different dc electric fields (shifted along vertical axis for clarity). The value of the applied dc electric field is given for each spectrum in units of $\mathrm{mV} / \mathrm{cm}$.

The line marked by an asterisk in Fig. 5a, and which is assigned to the $5 \rightarrow 41 p 5_{5}$ transition represents an exception. The $41 p 5_{5}$ Rydberg state is located above the O5 ionization threshold at $34160.15 \mathrm{~cm}^{-1}$ and is thus embedded in the $N^{+}=3$ ionization continua. The observation of this line in the RSR-TI spectrum is only possible if the lifetime of the $41 p 5_{5}$ Rydberg state is on the order of $1 \mu$ s or longer. No other members of the $n p 5_{5}$ Rydberg series with $n \leq 90$ and lying above the $N^{+}=3$ ionization threshold were observed in the RSR-TI spectra, indicating that their autoionization lifetimes are all much shorter than $1 \mu \mathrm{s}$, as a result of $p$ - $f$-interaction-mediated autoionization. The explanation for the abnormally long lifetime of the $41 p 5_{5}$ Rydberg state is that it is almost degenerate with the $23 f 7_{5}$ Rydberg state (calculated to lie only $0.0508 \mathrm{~cm}^{-1}$ below the $41 p 5_{5}$ Rydberg state). As a result of this near-degeneracy, the $p$ - $f$ interaction induces a strong admixture of $f 7_{5}$ character, which stabilizes the $41 p 5_{5}$ Rydberg state. The $n f 7_{5}$ Rydberg states in this region are long lived because rotational autoionization would necessitate a change of core rotational quantum number of -4 and is hindered. ${ }^{38}$

The electric-field dependence of the RSR-TI spectrum recorded near the $5 \rightarrow 41 p 5_{5}$ and $3 \rightarrow 38 p 3_{N}$ transitions is depicted in Fig. 10. Whereas the intensities of the $3 \rightarrow 38 p 3_{3,4}$ transitions do not vary as a function of the dc electric field, the line corresponding to the 
$5 \rightarrow 41 p 5_{5}$ transition disappears when dc electric fields larger than $52 \mathrm{mV} / \mathrm{cm}$ are applied. This observation can be explained by $\ell$ and $N$ mixing induced by the field. The largest field-induced interactions affecting the $41 p 5_{5}$ Rydberg state are those with $\Delta \ell= \pm 1$ and $\Delta N=0$ and autoionization is therefore expected to take place into the $d 3_{5}$ continuum. The interaction, and thus the autoionization rate, increases gradually with the magnitude of the electric field, which eventually leads to the disappearance of the $5 \rightarrow 41 p 5_{5}$ transition in the spectra recorded when fields of $\pm 78 \mathrm{mV} / \mathrm{cm}$ are applied. These results indicate that the $p$ - $f$ interaction is not the sole mechanism responsible for the autoionization lifetimes of the $n p N_{N=N^{+}}^{+}$and the $n p N_{N=N^{+}+1}^{+}$Rydberg series and that electric fields can also play an important role.

The measurement presented in Fig. 10 also allowed us to estimate the stray electric field in the excitation region: The intensity of the $5 \rightarrow 41 p 5_{5}$ transition measured when applying an electric field of $-26 \mathrm{mV} / \mathrm{cm}$ is smaller than when an electric field of $+26 \mathrm{mV} / \mathrm{cm}$ is applied, but corresponds approximately to the intensity measured when an electric field of $+52 \mathrm{mV} / \mathrm{cm}$ is applied. We therefore conclude that a dc electric field of $\sim 13 \mathrm{mV} / \mathrm{cm}$ is required to compensate the stray electric field $(-13 \mathrm{mV} / \mathrm{cm})$ present during laser excitation.

The joint effects of the $p-f$ interaction and $\ell$ mixing by electric fields on the autoionization dynamics of the triplet $n p$ Rydberg states around $n=100$ are illustrated by Fig. 11, which compares the PI and RSR-TI spectra recorded in regions Q3 and Q5 (see Fig. 2). The $n p 3_{2}$ and $n p 5_{4}$ series are not observed in these spectra because of rapid autoionization, as explained above. The calculated positions of the $n p 3_{3}$ and $n p 5_{5}$, the autoionization of which must be to an $f$ continuum in the absence of fields, are indicated as vertical dotted lines in Figs. 11a and b, respectively. Transitions to these states are strong in the PI spectra, but absent from the RSR-TI spectra, confirming the observation made for the $n p 7_{7}$ series (see Fig. 4d) that the $n p N_{N=N^{+}}^{+}$series have lifetimes significantly shorter than $2 \mu$ s around $n=100$. The calculated positions of the $n p N_{N=N^{+}+1}^{+}$Rydberg series are indicated by vertical dashed lines in Fig. 11. Whereas the transitions to the $n p 3_{4}$ and $n p 5_{6}$ Rydberg states have approximately the same intensities in the PI spectra, the lines corresponding to the transitions to the $n p 3_{4}$ Rydberg states are four times weaker in the RSR-TI spectra than those corresponding to the $n p 5_{6}$ Rydberg states.

These observations suggest that the $n p N_{N=N^{+}+1}^{+}$Rydberg states are longer lived than the $n p N_{N=N^{+}}^{+}$Rydberg states of the same principal quantum number. This difference may 


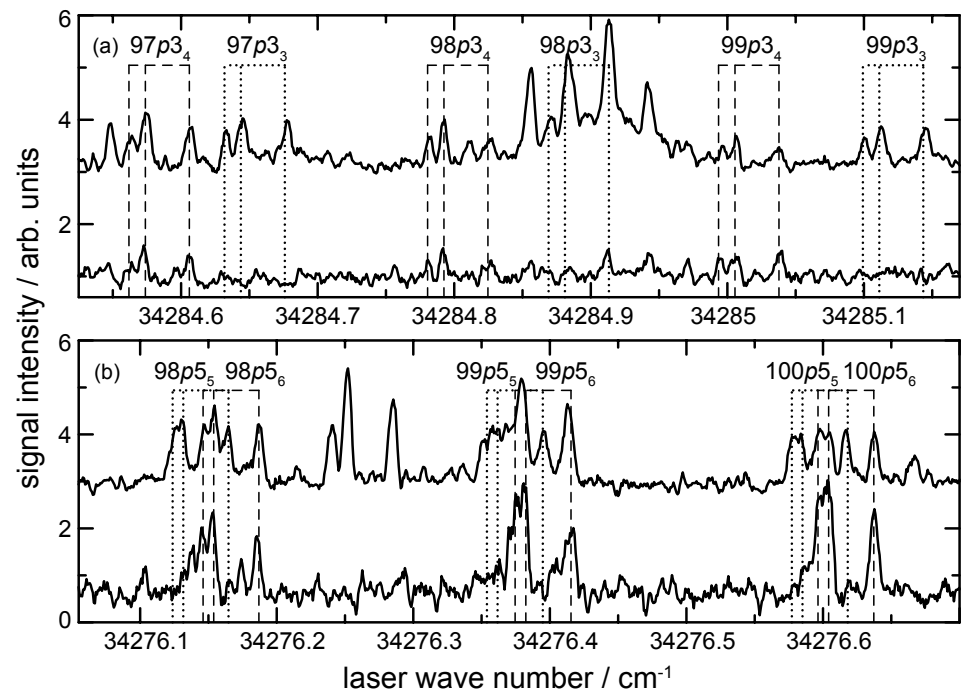

FIG. 11. Comparison between the PI spectra (top trace, shifted along the vertical axis for clarity) and the RSR-TI spectra (bottom trace) recorded (a) near the $3 \rightarrow 97-99 p 3_{N}$ transitions (region Q3) and (b) near the $5 \rightarrow 98-100 p 5_{N}$ transitions (region Q5). The calculated positions of the $N=N^{+}$and $N=N^{+}+1$ Rydberg states are indicated as vertical dotted and dashed lines, respectively.

originate from the $p$ - $f$ interaction but may also be explained by the influence of the stray electric fields of $13 \mathrm{mV} / \mathrm{cm}$ present in the photoexcitation region. The effect of the stray field on the $N=N^{+}$Rydberg states is to facilitate autoionization by inducing a $\Delta \ell=1, \Delta N=0$ interaction between the $n p 3_{3}\left(n p 5_{5}\right)$ Rydberg states and the $d 1_{3}\left(d 3_{5}\right)$ ionization continuum, as discussed in the context of Fig. 10 for the $41 p 5_{5}$ Rydberg state. The electric field necessary to induce such an interaction is smaller for Rydberg states around $n=100$, because the polarizability of Rydberg states scales as $n^{7}$. The lifetimes of the $n p 3_{4}$ Rydberg states are expected to be less affected by the stray field than the $n p 3_{3}$ Rydberg state. Indeed, the $n p 3_{4}$ Rydberg states cannot decay via a $\Delta \ell=1, \Delta N=0$ interaction because the $N^{+}=1, \ell=2$ ionization continuum does not possess any $N=4$ character. The fieldinduced decay must therefore proceed via a $\Delta \ell=1, \Delta N=1$ rather than a (stronger) $\Delta \ell=1, \Delta N=0$ interaction. The surprisingly long lifetime of the $n p 5_{6}$ Rydberg states around $n=100$ is a consequence of the fact that their effective quantum defects are almost zero (see Fig. 9c) and that, consequently, their positions coincide with the positions of the manifold of nonpenetrating $(\ell \geq 3)$ Stark states, to which they are coupled by the $p-f$ interaction. The stray field, though weak, therefore efficiently induces high- $\ell$ character to the $n p 5_{6}$ Rydberg states around $n=100$, which prolongs their lifetimes beyond $2 \mu$ s. 
TABLE VIII. Rotational energies of ${ }^{4} \mathrm{He}_{2}^{+}$in the $X^{+2} \Sigma_{u}^{+}\left(v^{+}=0\right)$ ground state relative to the $N^{+}=1$ level as determined in various studies. All values are given in units of $\mathrm{cm}^{-1}$.

\begin{tabular}{c|cccc}
\hline \hline$N^{+}$ & $\begin{array}{c}\text { Ref. 18 } \\
\text { (ZEKE) }\end{array}$ & $\begin{array}{c}\text { Ref. 18 } \\
\text { (MQDT fit) }\end{array}$ & $\begin{array}{c}\text { Ref. 7 } \\
(\text { ab initio })\end{array}$ & $\begin{array}{c}\text { Present work } \\
\text { (MQDT fit) }\end{array}$ \\
\hline 1 & 0 & 0 & 0 & $0^{\mathrm{a}}$ \\
3 & $70.943(6)$ & $70.918(3)$ & 70.936 & $70.937(3)$ \\
5 & $198.379(16)$ & $198.317(8)$ & 198.359 & $198.369(6)$ \\
7 & $381.858(31)$ & $381.761(16)$ & 381.822 & $381.822^{\mathrm{b}}$ \\
\hline \hline
\end{tabular}

a The term value of the $\left(v^{+}=0, N^{+}=1\right)$ ionization threshold relative to the hypothetical $\left(v^{\prime \prime}=0, N^{\prime \prime}=0\right)$ level of the $2 s \sigma a^{3} \Sigma_{u}^{+}$state of ${ }^{4} \mathrm{He}_{2}$ was determined to be $34316.387(10) \mathrm{cm}^{-1}$, which represents the average of the results of fit A $\left(34316.391 \mathrm{~cm}^{-1}\right)$ and fit $\mathrm{C}\left(34316.383 \mathrm{~cm}^{-1}\right)$.

b Fixed to the $a b$ initio value of Ref. 7 .

\section{CONCLUSIONS}

The term values of triplet $n p$ Rydberg states with $n>20$ converging to the $\left(v^{+}=\right.$ $\left.0, N^{+}=1,3,5,7\right)$ levels of the $X^{+2} \Sigma_{u}^{+}$ground state of ${ }^{4} \mathrm{He}_{2}^{+}$have been obtained by Rydbergstate-resolved threshold-ionization (RSR-TI) spectroscopy. By combining the data with spectroscopic data on low-lying triplet $n p$ and $n f$ Rydberg states from the literature ${ }^{22-29}$ a new set of energy- and $R$-dependent eigenquantum-defect parameters of multichannel quantum-defect theory was derived using a methodology established by Ch. Jungen and his coworkers $^{40,45-54}$. The MQDT model includes the effects of $p-f$ and rovibrational channel interactions and provides an accurate description of all triplet $n p$ and $n f$ Rydberg states of $\mathrm{He}_{2}$ with $v^{+} \leq 2$ and $N^{+} \leq 9\left(N^{+} \leq 19\right.$ for states with negative electronic parity). The root-mean-square deviation between observed and calculated term values was $0.005 \mathrm{~cm}^{-1}$ for the Rydberg states measured in this investigation $(n>20)$ and $0.2 \mathrm{~cm}^{-1}$ for the low- $n$ Rydberg states taken from the literature $(n=2-20)$. These deviations correspond to the experimental uncertainties of the respective measurements.

The adjusted eigenquantum-defect functions were combined with the potential energy function of the ground electronic state of $\mathrm{He}_{2}^{+}$from Ref. ${ }^{7}$ to derive the Born-Oppenheimer potential-energy functions and the leading adiabatic corrections of the triplet $n p$ Rydberg states of $\mathrm{He}_{2}$ with $n \leq 5$ in the range of internuclear distances $R=1.7-2.7 a_{0}$. It will be interesting in future to compare these results with potential-energy curves obtained by $a b$ initio quantum chemistry.

Rotational spectroscopy on ${ }^{4} \mathrm{He}_{2}^{+}$at an accuracy of up to $0.003 \mathrm{~cm}^{-1}$ was performed 
by extrapolating the experimental term values of triplet $n p$ Rydberg states in the range $n=87-110$ to their ionization limit using MQDT. The resulting positions of the lowest rotational levels of ${ }^{4} \mathrm{He}_{2}^{+}$are compared to previous results from the literature in Table VIII. The energy of the $\left(v^{+}=0, N^{+}=3\right)$ rotational level is in excellent agreement with the latest ab initio calculations ${ }^{7}$ and the value obtained by ZEKE spectroscopy. ${ }^{18}$ A deviation on the order of six standard deviations is found with the latest value extrapolated from the energies of Rydberg states. ${ }^{18}$ The reason for this deviation lies presumably in the neglect of $p-f$ and vibrational channel interactions in the MQDT model used in Ref. 18. For the energy of the $\left(v^{+}=0, N^{+}=5\right)$ rotational level of ${ }^{4} \mathrm{He}_{2}^{+}$we find a small deviation with the $a b$ initio calculations from Ref. 7. The deviation might result from systematic dc Stark shifts of the high- $n$ Rydberg states around $n=100$ used to extrapolate the rotational energies in the present study. Alternatively, it may reflect the neglect of quantum-electrodynamics corrections in the ab initio calculations of the rotational energies. Such corrections were found to be significant in $\mathrm{H}_{2} \cdot{ }^{61,62}$ Our result is in good agreement with, but more accurate than, the result obtained by ZEKE spectroscopy ${ }^{18}$ but deviates significantly from the latest value extrapolated from the energies of Rydberg states, ${ }^{18}$ a deviation we also attribute to the incomplete nature of the MQDT model used in Ref. 18.

The delayed pulsed-field ionization of Rydberg states in RSR-TI spectroscopy restricts the detection to long-lived Rydberg states only. The autoionization dynamics of the triplet $n p\left(v^{+}=0\right)$ Rydberg states with $N=N^{+}$and $N=N^{+}+1$ was found to be governed by the $p$ - $f$ interaction, which reduces the lifetimes to values below $2 \mu$ s for levels with $n \lesssim 90$. The $41 p 5_{5}$ Rydberg states has an exceptionally long lifetime of $>2 \mu$ s because of the $p-f$ interaction with the long-lived $23 f 7_{5}$ Rydberg state. At higher $n$ values, the stray electric field of $\sim 13 \mathrm{mV} / \mathrm{cm}$ induces $N$ - and $\ell$-mixing and governs the autoionization dynamics. The field-induced interactions can lead to shorter or longer lifetimes, depending on whether the interaction is with an ionization continuum or with long-lived Rydberg states.

\section{ACKNOWLEDGMENTS}

We dedicate this article to Christian Jungen (Laboratoire Aimé Cotton du CNRS, Orsay) and thank him for useful discussions and for allowing us to use his MQDT program. DS and FM also thank him and the Laboratoire Aimé Cotton for numerous research periods in 
Orsay. We thank Matthias Raunhardt for his help in the operation of the discharge used to generate metastable $\mathrm{He}_{2}$. This work was supported financially by the Swiss National Science Foundation under project 200020-146759 and by the European Research Council advanced grant program under project 228286 .

\section{REFERENCES}

${ }^{1}$ T. M. Miller, in CRC Handbook of Chemistry and Physics, 94th Edition, edited by W. M. Haynes (CRC Press/Taylor and Francis, Boca Raton, FL, Internet Version 2014).

${ }^{2}$ D. Z. Kandula, Ch. Gohle, T. J. Pinkert, W. Ubachs, and K. S. E. Eikema, Phys. Rev. Lett. 105, 063001 (2010).

${ }^{3}$ F. Grandinetti, Int. J. Mass Spectrom. 237, 243 (2004).

${ }^{4}$ F. Luo, G. C. McBane, G. Kim, C. F. Giese, and W. R. Gentry, J. Chem. Phys. 98, 3564 (1993).

${ }^{5}$ W. Schöllkopf and J. P. Toennies, Science 266, 1345 (1994).

${ }^{6}$ W. Cencek, , M. Przybytek, J. Komasa, J. B. Mehl, B. Jeziorski, and K. Szalewicz, J. Chem. Phys. 136, 224303 (2012).

${ }^{7}$ W.-C. Tung, M. Pavanello, and L. Adamowicz, J. Chem. Phys. 136, 104309 (2012).

${ }^{8}$ C. W. Bauschlicher, Jr., H. Partridge, and D. Ceperley, Chem. Phys. Lett. 160, 183 (1989).

${ }^{9}$ J. Ackermann and H. Hogreve, Chem. Phys. 157, 75 (1991).

${ }^{10}$ W. Cencek and J. Rychlewski, J. Chem. Phys. 102, 2533 (1995).

${ }^{11}$ A. Carrington, C. H. Pyne, and P. J. Knowles, J. Chem. Phys. 102, 5979 (1995).

${ }^{12}$ F. X. Gadéa and I. Paidarová, Chem. Phys. 209, 281 (1996).

${ }^{13}$ W. Cencek and J. Rychlewski, Chem. Phys. Lett. 320, 549 (2000).

${ }^{14}$ J. Xie, B. Poirier, and G. I. Gellene, J. Chem. Phys. 122, 184310 (2005).

${ }^{15}$ M. Stanke, D. Kędziera, S. Bubin, M. Molski, and L. Adamowicz, Phys. Rev. A 76, 052506 (2007).

${ }^{16}$ A. Ruzsinszky, J. P. Perdew, G. I. Csonka, O. A. Vydrov, and G. E. Scuseria, J. Chem. Phys. 126, 104102 (2007).

${ }^{17}$ N. Yu and W. H. Wing, Phys. Rev. Lett. 59, 2055 (1987).

${ }^{18}$ M. Raunhardt, M. Schäfer, N. Vanhaecke, and F. Merkt, J. Chem. Phys. 128, 164310 
(2008).

${ }^{19}$ L. Coman, M. Guna, L. Simons, and K. A. Hardy, Phys. Rev. Lett. 83, 2715 (1999).

${ }^{20}$ W. J. van der Zande and W. Ubachs, Phys. Rev. Lett. 84, 3212 (2000).

${ }^{21}$ K. Hardy and X. W. Wang, Phys. Rev. Lett. 84, 3213 (2000).

${ }^{22}$ D. S. Ginter, M. L. Ginter, and C. M. Brown, J. Chem. Phys. 81, 6013 (1984).

${ }^{23}$ D. S. Ginter and M. L. Ginter, J. Mol. Spectrosc. 82, 152 (1980).

${ }^{24}$ F. B. Orth and M. L. Ginter, J. Mol. Spectrosc. 64, 223 (1977).

${ }^{25}$ F. B. Orth and M. L. Ginter, J. Mol. Spectrosc. 61, 282 (1976).

${ }^{26}$ C. M. Brown and M. L. Ginter, J. Mol. Spectrosc. 40, 302 (1971).

${ }^{27}$ G. Herzberg and Ch. Jungen, J. Chem. Phys. 84, 1181 (1986).

${ }^{28}$ C. Focsa, P. F. Bernath, and R. Colin, J. Mol. Spectrosc. 191, 209 (1998).

${ }^{29}$ K. Kawaguchi, Y. Hama, and S. Nishida, J. Mol. Spectrosc. 232, 1 (2005).

${ }^{30}$ D. N. McKinsey, C. R. Brome, J. S. Butterworth, S. N. Dzhosyuk, P. R. Huffman, C. E. H. Mattoni, J. M. Doyle, R. Golub, and K. Habicht, Phys. Rev. A 59, 200 (1999).

${ }^{31}$ R. Seiler, Th. Paul, M. Andrist, and F. Merkt, Rev. Sci. Instr. 76, 103103 (2005).

${ }^{32}$ R. Seiler, U. Hollenstein, G. M. Greetham, and F. Merkt, Chem. Phys. Lett. 346, 201 (2001).

${ }^{33}$ R. Seiler, U. Hollenstein, T. P. Softley, and F. Merkt, J. Chem. Phys. 118, 10024 (2003).

${ }^{34}$ U. Hollenstein, R. Seiler, and F. Merkt, J. Phys. B: At. Mol. Opt. Phys. 36, 893 (2003).

${ }^{35}$ S. Gerstenkorn, J. Vergès, and J. Chevillard, Atlas du Spectre d'Absorption de la Molécule d'Iode: 11000-14000 $\mathrm{cm}^{-1}$ (Laboratoire Aimé-Cotton CNRS II, Orsay, 1982).

${ }^{36}$ D. Sprecher, J. Liu, Ch. Jungen, W. Ubachs, and F. Merkt, J. Chem. Phys. 133, 111102 (2010).

${ }^{37}$ J. Liu, E. J. Salumbides, U. Hollenstein, J. C. J. Koelemeij, K. S. E. Eikema, W. Ubachs, and F. Merkt, J. Chem. Phys. 130, 174306 (2009).

${ }^{38}$ C. R. Mahon, G. R. Janik, and T. F. Gallagher, Phys. Rev. A 41, 3746 (1990).

${ }^{39}$ F. Merkt, H. H. Fielding, and T. P. Softley, Chem. Phys. Lett. 202, 153 (1993).

${ }^{40}$ G. Herzberg and Ch. Jungen, J. Mol. Spectrosc. 41, 425 (1972).

${ }^{41}$ F. Merkt and T. P. Softley, Phys. Rev. A 46, 302 (1992).

${ }^{42}$ J. W. C. Johns and D. W. Lepard, J. Mol. Spectrosc. 55, 374 (1975).

${ }^{43}$ W. A. Chupka, J. Chem. Phys. 98, 4520 (1993).

${ }^{44}$ F. Merkt and R. N. Zare, J. Chem. Phys. 101, 3495 (1994). 
${ }^{45}$ Ch. Jungen and O. Atabek, J. Chem. Phys. 66, 5584 (1977).

${ }^{46}$ C. H. Greene and Ch. Jungen, Adv. At. Mol. Phys. 21, 51 (1985).

${ }^{47}$ S. C. Ross and Ch. Jungen, Phys. Rev. A 49, 4353 (1994).

${ }^{48}$ S. C. Ross and Ch. Jungen, Phys. Rev. A 49, 4364 (1994).

${ }^{49}$ S. C. Ross and Ch. Jungen, Phys. Rev. A 50, 4618 (1994).

${ }^{50}$ S. C. Ross, Ch. Jungen, and A. Matzkin, Can. J. Phys. 79, 561 (2001).

${ }^{51}$ Ch. Jungen, K. P. Huber, M. Jungen, and G. Stark, J. Chem. Phys. 118, 4517 (2003).

${ }^{52}$ A. Osterwalder, A. Wüest, F. Merkt, and Ch. Jungen, J. Chem. Phys. 121, 11810 (2004).

${ }^{53}$ M. Glass-Maujean and Ch. Jungen, J. Phys. Chem. A 113, 13124 (2009).

${ }^{54} \mathrm{Ch}$. Jungen, Elements of quantum defect theory in Handbook of High-resolution Spectroscopy, edited by M. Quack and F. Merkt (John Wiley \& Sons, Ltd., 2011).

${ }^{55}$ D. Sprecher, Ch. Jungen, and F. Merkt, J. Phys. Chem. A 117, 9353 (2013).

${ }^{56}$ L. Wolniewicz, J. Mol. Spectrosc. 174, 132 (1995).

${ }^{57}$ W. Kołos and J. Rychlewski, J. Mol. Spectrosc. 177, 146 (1996).

${ }^{58}$ G. Staszewska and L. Wolniewicz, J. Mol. Spectrosc. 198, 416 (1999).

${ }^{59}$ G. Staszewska, J. Phys. Chem. A 105, 2308 (2001).

60 See supplementary material at http:// for tables containing experimental term values of triplet $n p$ Rydberg states of $\mathrm{He}_{2}$ taken from the literature and their comparison to MQDT calculations.

${ }^{61}$ J. Komasa, K. Piszczatowski, G. Łach, M. Przybytek, B. Jeziorski, and K. Pachucki, J. Chem. Theory Comput. 7, 3105 (2011).

${ }^{62}$ E. J. Salumbides, G. D. Dickenson, T. I. Ivanov, and W. Ubachs, Phys. Rev. Lett. 107, 043005 (2011). 